\title{
Comprehensive molecular biomarker identification in breast cancer brain metastases
}

\author{
Hans-Juergen Schulten ${ }^{1 *} \mathbb{D}$, Mohammed Bangash², Sajjad Karim", Ashraf Dallol ${ }^{1}$, Deema Hussein ${ }^{3}$, \\ Adnan Merdad ${ }^{4}$, Fatma K. Al-Thoubaity ${ }^{4}$, Jaudah Al-Maghrabi i,6, Awatif Jamal' ${ }^{5}$, Fahad Al-Ghamdi ${ }^{5}$, \\ Hani Choudhry' ${ }^{7}$ Saleh S. Baeesa ${ }^{2}$, Adeel G. Chaudhary ${ }^{1}$ and Mohammed H. Al-Qahtani ${ }^{1}$
}

\begin{abstract}
Background: Breast cancer brain metastases (BCBM) develop in about 20-30\% of breast cancer (BC) patients. BCBM are associated with dismal prognosis not at least due to lack of valuable molecular therapeutic targets. The aim of the study was to identify new molecular biomarkers and targets in BCBM by using complementary state-of-the-art techniques.
\end{abstract}

Methods: We compared array expression profiles of three BCBM with 16 non-brain metastatic $B C$ and 16 primary brain tumors (prBT) using a false discovery rate (FDR) $p<0.05$ and fold change $(F C)>2$. Biofunctional analysis was conducted on the differentially expressed probe sets. High-density arrays were employed to detect copy number variations (CNVs) and whole exome sequencing (WES) with paired-end reads of $150 \mathrm{bp}$ was utilized to detect gene mutations in the three BCBM.

Results: The top 370 probe sets that were differentially expressed between BCBM and both $B C$ and prBT were in the majority comparably overexpressed in BCBM and included, e.g. the coding genes BCL3, BNIP3, BNIP3P1, BRIP1, CASP14, CDC25A, DMBT1, IDH2, E2F1, MYCN, RAD51, RAD54L, and VDR. A number of small nucleolar RNAs (snoRNAs) were comparably overexpressed in BCBM and included SNORA1, SNORA2A, SNORA9, SNORA10, SNORA22, SNORA24, SNORA30, SNORA37, SNORA38, SNORA52, SNORA71A, SNORA71B, SNORA71C, SNORD13P2, SNORD15A, SNORD34, SNORD35A, SNORD41, SNORD53, and SCARNA22. The top canonical pathway was entitled, role of BRCA1 in DNA damage response. Network analysis revealed key nodes as Akt, ERK1/2, NFkB, and Ras in a predicted activation stage. Downregulated genes in a data set that was shared between BCBM and prBT comprised, e.g. BC cell line invasion markers JUN, MMP3, TFF1, and HAS2. Important cancer genes affected by CNVs included TP53, BRCA1, BRCA2, ERBB2, IDH1, and IDH2. WES detected numerous mutations, some of which affecting BC associated genes as CDH1, HEPACAM, and LOXHD1.

Conclusions: Using complementary molecular genetic techniques, this study identified shared and unshared molecular events in three highly aberrant BCBM emphasizing the challenge to detect new molecular biomarkers and targets with translational implications. Among new findings with the capacity to gain clinical relevance is the detection of overexpressed snoRNAs known to regulate some critical cellular functions as ribosome biogenesis.

Keywords: Breast cancer brain metastases, Whole transcript array profiling, Pathway and network analysis, snoRNAs, Copy number variations, Whole exome sequencing

\section{Background}

It is estimated that $20-30 \%$ of all breast cancer $(B C)$ develop BC brain metastases (BCBM) [1]. Recent

\footnotetext{
*Correspondence: hschulten@kau.edu.sa

${ }^{1}$ Center of Excellence in Genomic Medicine Research, King Abdulaziz University, Jeddah, Saudi Arabia

Full list of author information is available at the end of the article
}

studies indicate that BCBM is highest in ERBB2 (HER2) and triple negative $\mathrm{BC}$ (TNBC) with an incidence of $20-50 \%$ [2-5]. The high incidence of BCBM in ERBB2 tumors possibly could be attributed to ERBB2 targeted treatment that leads to an initially increased survival $[6,7]$. Mean time between primary $\mathrm{BC}$ and $\mathrm{BCBM}$ is approximately 35 months and main associated variables are tumor size and lymph node metastasis $[8$, 
9]. Approximately $30 \%$ of $\mathrm{BC}$ patients reveal to have a BCBM at autopsy $[10,11]$. The majority of patients receive a multimodality therapy approach that may include treatment with an anti-Human ERBB2 therapeutic antibody in ERBB2 positive tumors and hormonal therapy in ER and/or PR positive tumors [9]. ERBB2 amplifications and mutations are frequently found in $\mathrm{BC}$ and corresponding BCBM [12]. Targeted therapy options for triple negative $B C B M$, which harbor frequently $B R C A 1$ and $B R C A 2$ aberrations, are currently not available in clinical practice. To improve treatment of BCBM, a number of advanced therapy trials and new targeted therapy options are emerging [1, 13-15]. Molecular targets include e.g. ERBB2, EGFR, VEGFR, PARP, and the mTOR and CDK-4/6 pathways.

The brain metastatic process is a multistep sequence involving migration, intravasation, circulation, arrest, extravasation, and settlement/invasion of the brain microenvironment $[5,16,17]$. Especially, the blood brain barrier (BBB) is highly selective for both tumor cells and drug therapeutics to enter the brain microenvironment. Consistent with this, it has been demonstrated that brain metastatic lesions have a monoclonal or predominantly monoclonal origin [5, 18-21]. This implicates that a brain metastasis shares common aberrations with the metastatic ancestor cell while subsequent evolving aberrations may only be present in brain metastatic subclones. A support for this may be the fact that TP53 mutations are likely to be more frequent in BCBM compared to BC (59\% vs. 39\%) [22].

Molecules and molecular mechanisms that control and regulate critical steps of the brain metastatic process are complex and subject of several studies. In vitro assays demonstrated that ERBB2-ERBB3 dimers promote $\mathrm{BBB}$ transendothelial migration fostered by a chemotaxic signal of their ligand NRG1 [23]. In brain and lung metastatic BC, COX2, EGFR, and HBEGF have been identified as promoting factors of extravasation through nonfenestrated blood vessels and of subsequent colonization [24]. An in vivo study demonstrated that brain metastatic cancer cells interact with the brain microenvironment to promote metastases [25]. Upregulated genes that support establishment of brain metastases include CXCR4, PLLP, TNFSF4, VCAM1, SLC8A2, and SLC7A11. Co-culture experiments demonstrated that $\mathrm{PCDH} 7$ directly interacts with GJA1, both which are known to be expressed in TNBC with brain metastatic behavior, to assemble functional gap junctions between cancer cells and astrocytes resulting in promoting brain metastasis via a paracrine activation loop [26]. A neuronal lineage cell reprogramming expression signature including upregulation of SNAP25, SNAP91, and BSN has been detected in xenograph brain tumors originating from human cell lines including a breast cell line with a preference to metastasize to the brain [27].

In the present study, we used complementary techniques to comprehensively analyze the tumor genetics of three BCBM with a focus to identify new molecular biomarkers and targets. We utilized for expression analysis whole transcript arrays that cover on average each exon of a gene with a probe. A number of studies have investigated expression profiles related to $\mathrm{BCBM}$ or to different steps of the brain metastatic process using various kinds of samples/model systems, methodologies/ techniques, and comparison groups rendering it difficult to identify common gene expression signatures $[22$, 24, 25, 28-34].

\section{Methods}

Tumor samples

Tumor samples from three consecutive BCBM, Jed $81_{-}$ MT, Jed82_MT, and Jed89_MT, were derived from patients who were treated surgically in 2015 at the King Abdulaziz University Hospital, Jeddah. Histopathological diagnosis was performed on established criteria. Age of patients at time of BCBM surgery was 60 years for Jed81_MT, 32 years for Jed82_MT, and 56 years for Jed89_MT, respectively. Time period between primary BC and BCBM was 13 years for Jed81_MT, 10 months for Jed82_MT, and 2 years for Jed89_MT. Other sites of distant metastases were reported for Jed81_MT. The three $\mathrm{BCBM}$ were classified as grade III tumors according to the Nottingham grading system. The generated array data set of the 35 samples from the core analysis has been deposited at the Gene Expression Omnibus (GEO) under Accession Number GSE100534 including basic demographic and histopathological data of each case. This GEO submission comprises samples of BC and primary brain tumors (prBT) which were previously included in GEO submissions GSE36295, GSE66463, and/or GSE77259.

\section{Sample selection for array expression study}

Sample selection criterion was to identify probe sets that are significant to the brain metastatic process. The selection procedure was carried out by analysis of variance (ANOVA) using a $p<0.05$ and fold change (FC) $>2$ as described earlier [35] in order to select those samples which have the comparably lowest numbers of significantly differentially expressed probe sets with the three BCBM. Therefore, in the core analysis we established the expression profiles of the three $\mathrm{BCBM}$ in relation to the expression profiles of 16 non-brain metastatic $B C$ and 16 prBT. The $16 \mathrm{BC}$ samples were selected from 45 cases of a previous BC study [36]. No histopathologically confirmed 
brain metastases were recorded for the $16 \mathrm{BC}$ in the available reports. Of the $16 \mathrm{BC}$, two were grade $\mathrm{I}$, four were grade II, one was grade II/III, six were grade III, and three were ungraded. Routine immunohistochemistry (IHC) staining for ERBB2 revealed score 0 in three, $1+$ in five, $2+$ in one, and $3+$ in three BC. For four BC no IHC scores for ERRB2 were available. The 16 prBT all of meningioma histology, were selected from 56 brain tumor samples of different histological types and for which array expression data were available at our repository. Of the 16 prBT, 12 were WHO grade I, three were grade II, and one was grade III. One grade II prBT was brain invasive and another grade II prBT was a recurrence.

\section{RNA and array expression processing}

Native tumor specimens for array expression analysis were transiently stored in RNALater (Qiagen, Hilden, Germany). Isolation of total RNA and array sample processing were performed as described earlier [37, 38]. In brief, the Agilent 2100 Bioanalyzer (Agilent Technologies, Palo Alto, CA) was employed to assess RNA integrity and integrity number was $>5$ in the samples used for differential expression analysis. RNA concentration was determined by using the NanoDrop ND-1000 spectrophotometer (NanoDrop Technologies, Wilmington, DE). All RNA samples were processed using the Ambion WT Expression Kit (Life Technologies, Austin, TX), the GeneChip WT Terminal Labeling and Controls Kit (Affymetrix, Santa Clara, CA), and the Affymetrix GeneChip Hybridization, Wash and Stain Kit. Samples were hybridized for $17 \mathrm{~h}$ to Affymetrix Human Gene 1.0 ST GeneChip arrays which interrogate with 764,885 probes 36.079 annotated reference sequences (NCBI build 36). On average, each exon of a gene is interrogated with one probe enabling to analyze expression data on the exon level [39]. The arrays were scanned on a GeneChip Scanner 3000 7G. Probe cell intensity data (CEL files) were generated by the GeneChip Command Console Software (AGCC).

\section{Array expression analysis}

The CEL files were imported to Partek Genomics Suite version 6.6 (Partek Inc., Chesterfield, MO) using default settings. QC metrics tables and QC graphical reports served as quality assessment of array expression experiments. The lists of differentially expressed probe sets were generated by ANOVA using either a $p<0.05$ and $\mathrm{FC}>2$ or using, where indicated, the more stringent criterion of the false discovery rate (FDR) $p$-value (step-up method) $<0.05$ and FC $>2$. Principal component analysis was utilized to illustrate overall variance in gene expression between samples or groups of samples. Average linkage hierarchical clustering was performed by using Spearman's correlation as a similarity matrix. Venn diagrams were generated to display genes that intersect or non-intersect between groups of differentially expressed probe sets. The gene ontology (GO) enrichment tool was employed in the gene expression workflow to group significantly expressed genes into functional categories. The gene enrichment score utilizes the Fisher`s exact test to determine the level of differential gene expression in a functional category. Alternative splicing analysis was applied to identify samples with differentially expressed exons.

\section{Functional network and pathway analysis}

Biological significance of expression data was interpreted by using the Ingenuity Pathways Analysis software (IPA; build version 338830 M) (Ingenuity Systems, Redwood City, CA) that uses the Ingenuity Knowledge Base as a reference data set. Direct and indirect molecular relationships were included in the analysis settings. Significance of relationships between analyzed data set molecules and functional frameworks prebuilt or generated de novo by IPA was indicated by Fisher's exact test $p$-values. The Molecule Activity Predictor was employed to predict expression effects/coherence of expression effects of a molecule on other pathway or network molecules. The canonical pathway workflow was employed to identify molecules from the uploaded data set that are coexpressed in a directional, up- to downstream, pathway. Network analysis was employed to explore significance of fit between molecules of the uploaded data set and networks related to specific diseases and functions. The percentage and number of uploaded molecules matching to molecules of a canonical pathway are a measure for its significance, expressed as a score. Upstream analysis was employed to explain how differences in target gene expression are effected by upstream regulators. The activation $\mathrm{z}$-score predicts the activation states of regulators. Regulator effects analysis was utilized to explain which regulators target differentially expressed genes from the uploaded data set and which kind of downstream effects, i.e. diseases and/or functions are associated. In how far a generated network is consistent with the knowledge base, i.e. either activated or inactivated, is scaled by a consistency score.

\section{Array copy number variation (CNV) analysis}

Array CNV analysis of the three BC brain metastases was performed in duplicate for each case using the CytoScan Reagent Kit according to the manufacturer's assay protocol (Affymetrix). In brief, tumor DNA from formalin-fixed and paraffin-embedded (FFPE) material was extracted by using the QIAamp DNA FFPE Tissue Kit (Qiagen, Hilden, Germany). The DNA processing steps included DNA restriction, adaptor ligation, PCR amplification 
and subsequent purification, quantification, fragmentation, and labeling of the PCR products. The hybridization mixtures containing the processed DNA samples were hybridized for $17 \mathrm{~h}$ at $50{ }^{\circ} \mathrm{C}$ and $60 \mathrm{rpm}$ to Cytoscan HD arrays. The array type interrogates the genome (build HG19) with 750,000 SNP probes and 1.9 million non-polymorphic probes. Subsequently, the arrays were washed stringently, then scanned on a GeneChip Scanner 3000 7G and the GeneChip Command Console Software (AGCC) was utilized to generate probe cell intensity data. Using default parameters the Chromosome Analysis Suite (ChAS) 3.1 was utilized to analyze genomic aberrations including copy number gains and losses, and mosaicisms. The ChAS software utilized NetAffx Genomic Annotation file NA33.1 (build HG19) which contains updated content from DGV, OMIM, and RefSeq. The reported aberrations are from overlapping regions of duplicate experiments.

\section{Immunohistochemistry}

Antibodies (Dako Denmark A/S, Glostrup, DK and Ventana Medical Systems, Tucson, AZ) employed for IHC of the three BCBM consisted of GFAP clone GF2, ERBB2 clone 5B5, and MKI67 clone MIB-1. Quantitative image analysis for proliferation marker MKI67, which is immunoreactive in the late $\mathrm{G} 1, \mathrm{~S}, \mathrm{G} 2$, and $\mathrm{M}$ phases of the cell cycle, was performed on at least 10 high power field images using the ImmunRatio application [40]. Measure of variability was expressed as standard deviation. Four $\mu \mathrm{m}$ sections of formalin-fixed and paraffin-embedded specimens were processed on an automated immunostainer (BenchMark XT, Ventana Medical Systems) according to the manufacturer's protocols and utilizing the ultraView Universal DAB Detection Kit for detection.

\section{Whole exome sequencing (WES)}

Exome capturing was performed using the Nextera Rapid Capture Expanded Exome Kit according to the manufacturer's protocol (Illumina, San Diego, CA). This application covers $62 \mathrm{Mb}$ of coding exons, UTRs, and regions of miRNAs. In brief, 50 ng FFPE DNA template from each of the BCBM were tagmented by using the TDE1 enzyme for DNA cleavage and adaptor ligation. Unique index adapters were added to each of the tagmented DNA samples that were subsequently amplified. The generated DNA libraries were purified and then pooled and hybridized to expanded exome oligos. The DNA library was captured by streptavidin magnetic beads. Hybridization and capturing steps were repeated and the enriched DNA library was subsequently amplified. Size distribution of DNA library fragments was analyzed using a high sensitivity DNA chip on an Agilent 2100 Bioanalyzer. Finally, utilizing the NextSeq 500 High Output v2 kit, a denatured aliquot of the DNA library was sequenced on an Illumina NextSeq 500 platform with paired-end reads of $150 \mathrm{bp}$ according to the manufacturer's protocol. FASTQ files were generated by the Illumina BaseSpace Hub. The FASTQ files were analysed using the Galaxy usegalaxy.org server [41] employing a number of bioinformatics workflow tools. For text manipulation the concatenate data sets tail-to-head (cat) (Galaxy 0.1.0) and for format conversion the FASTQ Groomer were utilized (Galaxy 1.0.4) [42]. Sequence mapping was performed with BWA for Illumina (Galaxy 1.2.3) using build HG19 canonical as reference genome [43]. FreeBayes bayesian genetic variant detector (Galaxy 1.0.2.29-3) was employed to detect polymorphisms and indels in the target region defined by the corresponding BED file [44]. The generated VCF files were uploaded to the Illumina BaseSpace Hub and variants were annotated and classified according to their biological significance using the VariantStudio App 1.0.0. Major filter settings included read depth $>10$, no reported global and population frequencies (de facto $=0$ ), and variant calling of mutations, comprising frameshift, stop gained or lost, initiator codon, inframe insertion or deletions, splice, and missense mutations, the latter if scored towards a deleterious and/or damaging variant by SIFT and Polyphen bioinformatic tools [45]. To minimize possible false positive results, the quality score was set on $>100$ excluding indicated mutations with quality scores between 0 and 100. A number of mutations were listed in the Single Nucleotide Polymorphism Database (dbSNP) [46] and/or in the Catalogue Of Somatic Mutations In Cancer (COSMIC) [47].

\section{Mutational analysis}

Mutational analysis was performed on FFPE DNA templates with minor modification according to our standard protocols [48]. PCR and sequencing primers are listed in Table 1. For direct sequencing, the purified PCR products

Table 1 Primer sequences used for conventional sequencing

\begin{tabular}{llll}
\hline Gene & Forward primer sequence $\left(\mathbf{3}^{\prime} \mathbf{-} \mathbf{5}^{\prime} \mathbf{)}\right.$ & Reverse primer sequence $\mathbf{( 3}^{\prime} \mathbf{- \mathbf { 5 } ^ { \prime } )}$ & Product $\mathbf{s i z e}(\mathbf{b p})$ \\
\hline LOXHD1 & AACACCTATGAGGTTCAGG & GTTGGACTTGTCTGACTTC & 134 \\
ERBB4 & AACCTGGAGATAACCAGC & CAAGGCATATCGATCCTC & 169 \\
CASP7 & TCGCTTTGGGTCTTCCA & TGCAGTTACCGTTCCCAC & 213 \\
\hline
\end{tabular}


were subjected to cycle sequence reactions using the BigDye Terminator V3.1 Cycle Sequencing kit (Applied Biosystems, Foster City, CA). Purified sequencing products were finally resolved by capillary electrophoresis on an ABI PRISM 3130 Sequencer.

\section{Results}

Genes differentially expressed in BCBM vs. $\mathrm{BC}$ and prBT

We performed an expression array study to establish expression profiles of three BCBM that differentiate them from those of 16 selected $\mathrm{BC}$ and 16 selected prBT. Similarity of expression profiles of all 35 samples is illustrated by a distance related matrix in a principal component analysis (PCA) 3D scatter plot showing that the three BCBM define a separate cluster between $\mathrm{BC}$ and prBT (Fig. 1). From the intersecting area of the two comparison groups BCBM vs. $\mathrm{BC}$ and $\mathrm{BCBM}$ vs. prBT, a compilation of 370 differentially expressed probe sets (FDR $p<0.05$ and FC $>2$ ) was established that primarily distinguishes the three BCBM from both, $\mathrm{BC}$ and prBT (Fig. 2; Additional file 1). Hierarchical cluster analysis on the 370 probe sets illustrates separate clustering of the three sample groups (Fig. 3). The 370 probe sets were

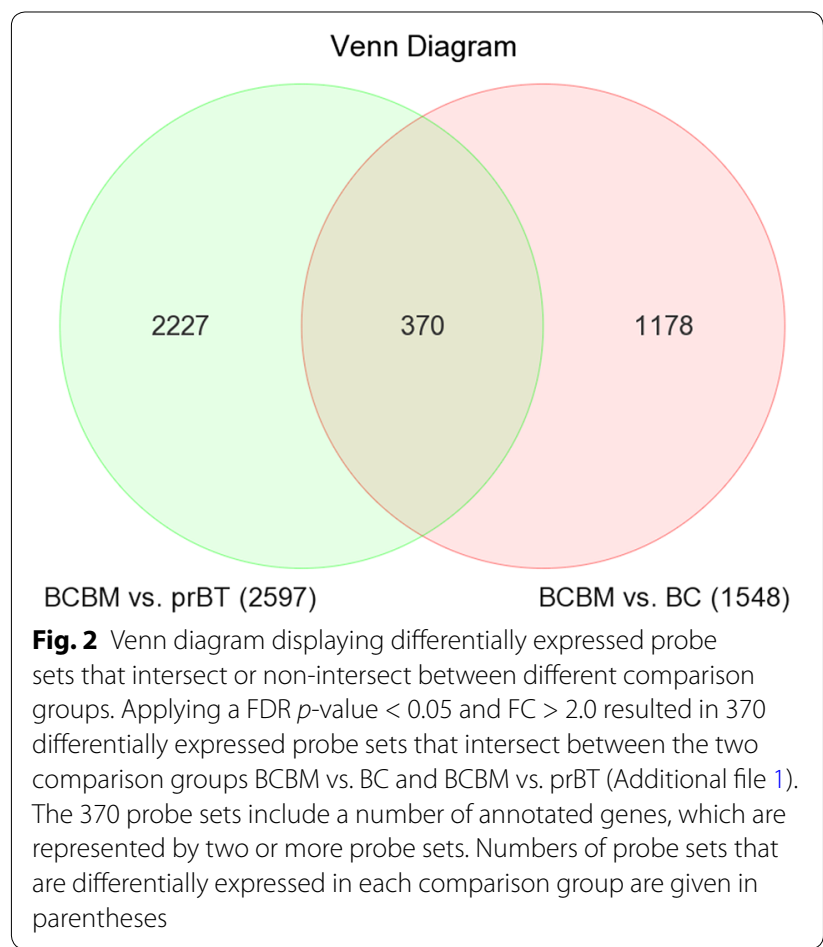

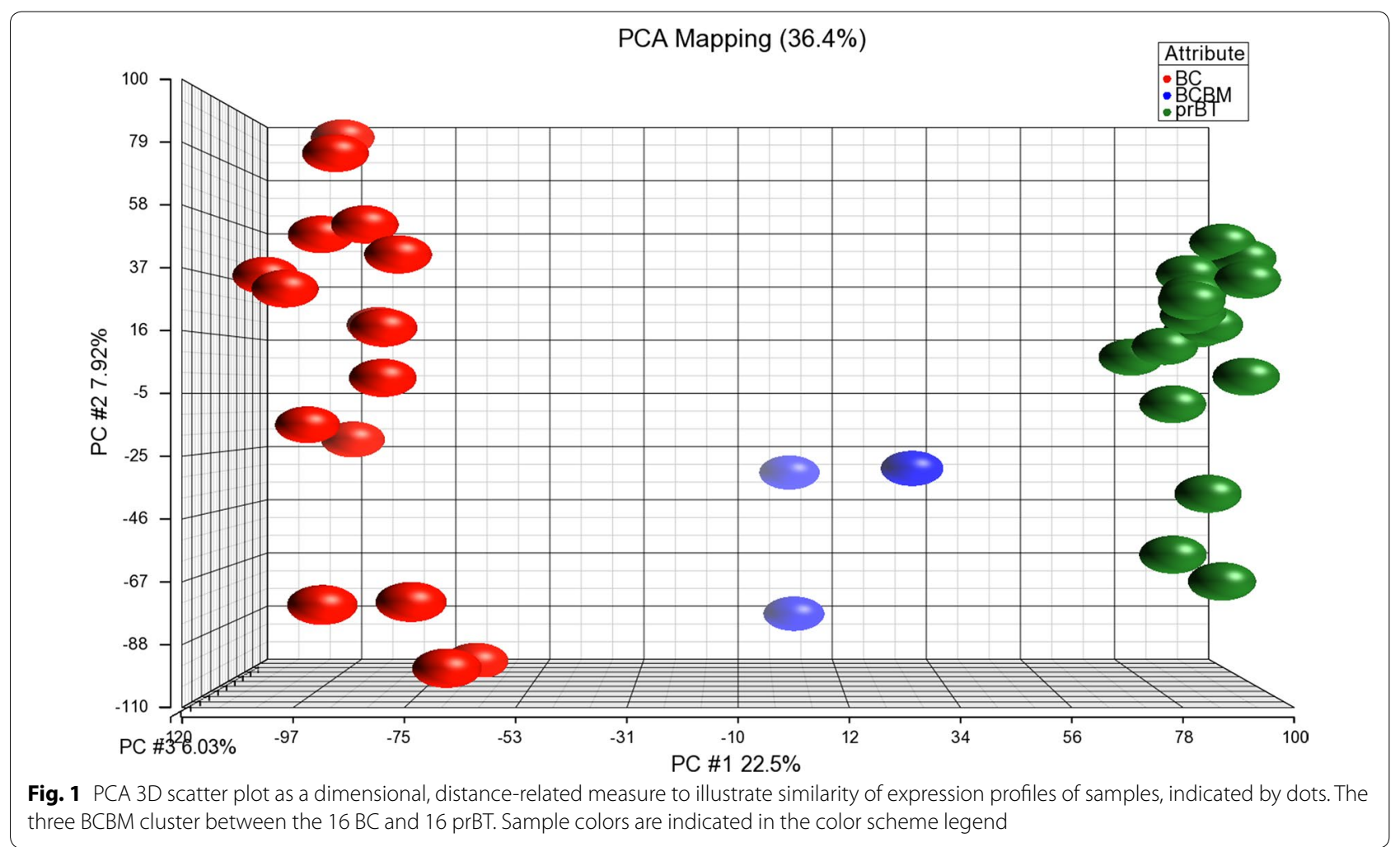




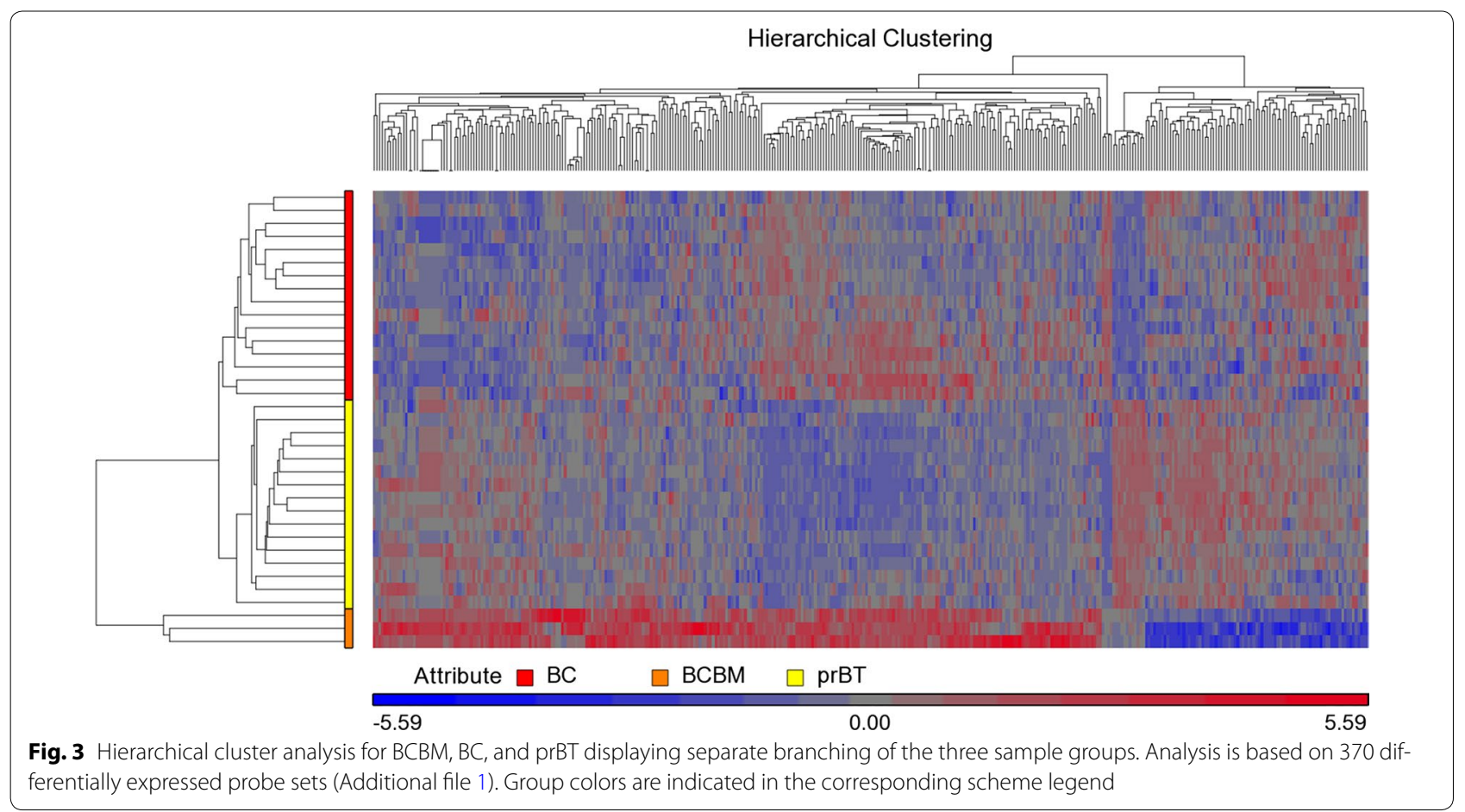

in the majority upregulated in the three $\mathrm{BCBM}$ when compared to BC ( $76 \%$ vs. $24 \%$ ) or compared to $\mathrm{prBT}$ (74\% vs. 26\%). Upregulated genes include, e.g. $B C L 3$, BNIP3, BNIP3P1, BRIP1, CASP14, CCNE2, CDC25A, CDC45, DMBT1, E2F1, EIF4EBP1, HILPDA, IDH2, MRPL13, MT-TK, MYCN, PPP1R14A, PPP1R1B, RAB3D, RAD51, RAD54L, RASGRF1, RRAGD, and VDR. Downregulated genes include, e.g. ARHGAP24, C1S, CDON, CLK1, DRAM1, GJA1, JAK2, KITLG, RABGAP1L, and RASSF8. A majority of non-coding RNAs were represented by small nucleolar RNAs (snoRNAs) that included SNORA1, SNORA2A, SNORA9, SNORA10, SNORA22, SNORA24, SNORA30, SNORA37, SNORA38, SNORA52, SNORA71A, SNORA71B, SNORA71C, SNORD13P2, SNORD15A, SNORD34, SNORD35A, SNORD41, SNORD53, and SCARNA22. Other non-coding RNAs comprised a number of small nuclear RNAs (snRNAs) as, e.g. RNU4ATAC18P, RNU6-1199P, and RNU6-447P. All snoRNAs and the vast majority of snRNAs were comparably overexpressed in BCBM. The long noncoding RNA $T E R C$ was same like comparably upregulated in BCBM. Expression of a number of cancer associated genes was studied on the exon level to assess possible exon splicing events that are not detected on the gene level. These genes comprise BRCA1, BRCA2, ERBB2, TP53, ESR1, PGR, SNORD116-4, MKI67, VDR, and BCL3 (Additional file $2 \mathrm{~A}-\mathrm{J})$.

\section{Biofunctional prediction analysis}

Gene ontology (GO) enrichment analysis on the 370 probe sets identified functional groups that were significantly overrepresented in different GO categories (Fig. 4). In the cellular component domain, the categories organelle, organelle part, and synapse part were prevalent. In the molecular function domain, the most significantly related categories were molecular function regulator, catalytic activity, and translation regulator activity. In the biological process domain, the prevalent categories were single-organism process, cellular component organization or biogenesis, and cellular process. Two of the top canonical pathways were entitled, Role of BRCA1 in DNA damage response (Fig. 5) and estrogen-mediated S-phase entry. Other top pathways were involved in biosynthesis of inositol phosphates and degradation of phosphoinositide (Table 2). The top three networks based on the 370 probe sets and merged in Fig. 6 were related to cell cycle, DNA replication, recombination, and repair, cellular assembly and organization, connective tissue and developmental disorders, gastrointestinal and inflammatory diseases, and inflammatory response (Table 2). A number of integrative key nodes including Akt, ERK1/2, NFkB, and Ras were in a predicted activation stage. An upstream regulator network was compiled including the predicted activated upstream regulators E2f, ERBB2, IL6, HGF, and RABL6, 


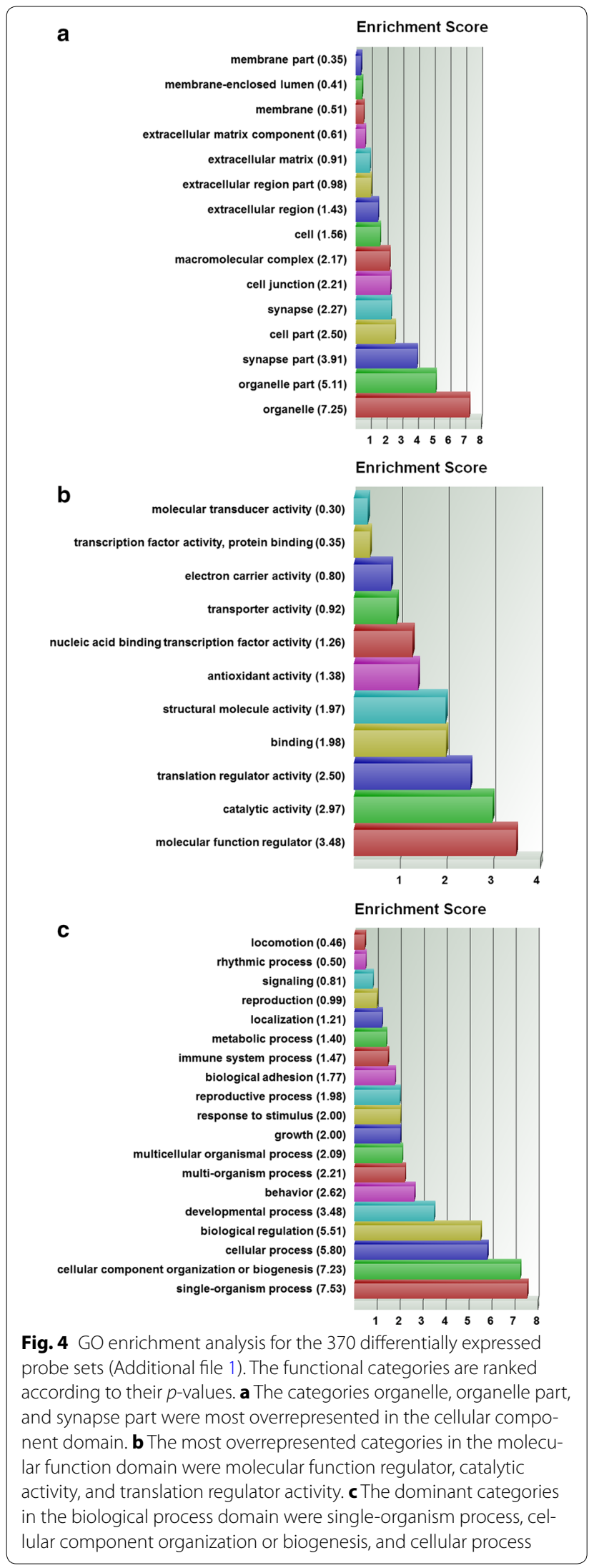

and the predicted inhibited upstream regulators let7, TP53, and CDKN2A (Fig. 7). E2f was the principal regulator effector on the four upregulated molecules RAD51, E2F1, ORC1, and CDC25A causing that the associated function entitled, metabolisms of DNA, was upregulated (Fig. 8).

\section{Genes and biofunctions shared between BCBM and prBT}

To identify candidate genes that may support the brain metastasis to adapt and expand in the brain microenvironment, we selected in our data compilation for those probe sets that intersect between the comparison groups $\mathrm{BCBM}$ vs. $\mathrm{BC}$ and $\mathrm{BC}$ vs. prBT (FDR $p<0.05$ and $\mathrm{FC}>2.0$ ). In addition, we excluded from the intersecting probe sets those that were included in the list of 585 probe sets derived from the intersection region of the two comparison groups $\mathrm{BCBM}$ vs. $\mathrm{BC}$ and $\mathrm{BCBM}$ vs. $\operatorname{prBT}(p<0.05$ and $\mathrm{FC}>2.0)$. We obtained a list of 643 probe sets that were in the majority upregulated $(\sim 60 \%)$ in BCBM compared to BC. Upregulated genes include, e.g. ATMIN, EIF4A1P2, EIF4B, CCNK, GNG10, HIGD2A, MALAT1, NCS1, NDRG1, PDPK1, RAB3A, RAB7A, RRAS, SOD3, and TIMP3. Downregulated genes include, e.g. BRMS1, CRLF3, FOSB, GREB1L, GPR141, JUN, MMP14, PARP9, PDCD4, PI4K2B, RAB27A, SOD1, and $V E G F C$. In addition, snoRNAs comprised about $7 \%$ of the probe sets and the vast majority were comparably upregulated. The top canonical pathway was entitled EIF2 signaling, containing mostly ribosomal proteins, i.e. RPL5, RPL7A, RPL13A, RPL36, RPL22, RPL31, RPL18A, RRAS, RPS14, RPL27A, ATF3, and RPL23A (Table 2). The top three networks based on the 643 probe sets that were common between BCBM and prBT and merged in Fig. 9 were related to cancer, cell death and survival, organismal injury and abnormalities, hereditary disorder, neurological disease, carbohydrate and drug metabolism, and molecular transport (Table 2). Il1B was the top regulator effector on the four downregulated, invasion related molecules JUN, MMP3, TFF1, and HAS2 (Fig. 10). Consequently, the associated function entitled, invasion of breast cell lines was downregulated implying that the invasive behavior of $\mathrm{BCBM}$ has been reprogrammed when compared to $\mathrm{BC}$.

\section{Array CNV analysis}

Array $\mathrm{CNV}$ analysis was performed in duplicate for each sample revealing highly aberrant genomic profiles of all three BCBM. Important cancer genes affected by CNVs include TP53, BRCA1, BRCA2, ERBB2, IDH1, and IDH2 (Table 3). Mosaic-loss of the chromosomal region that comprises TP53 was found in all three BCBM and may indicate a late cytogenetic event that is only present in subclones. Either $B R C A 1$ or $B R C A 2$ were affected in all 


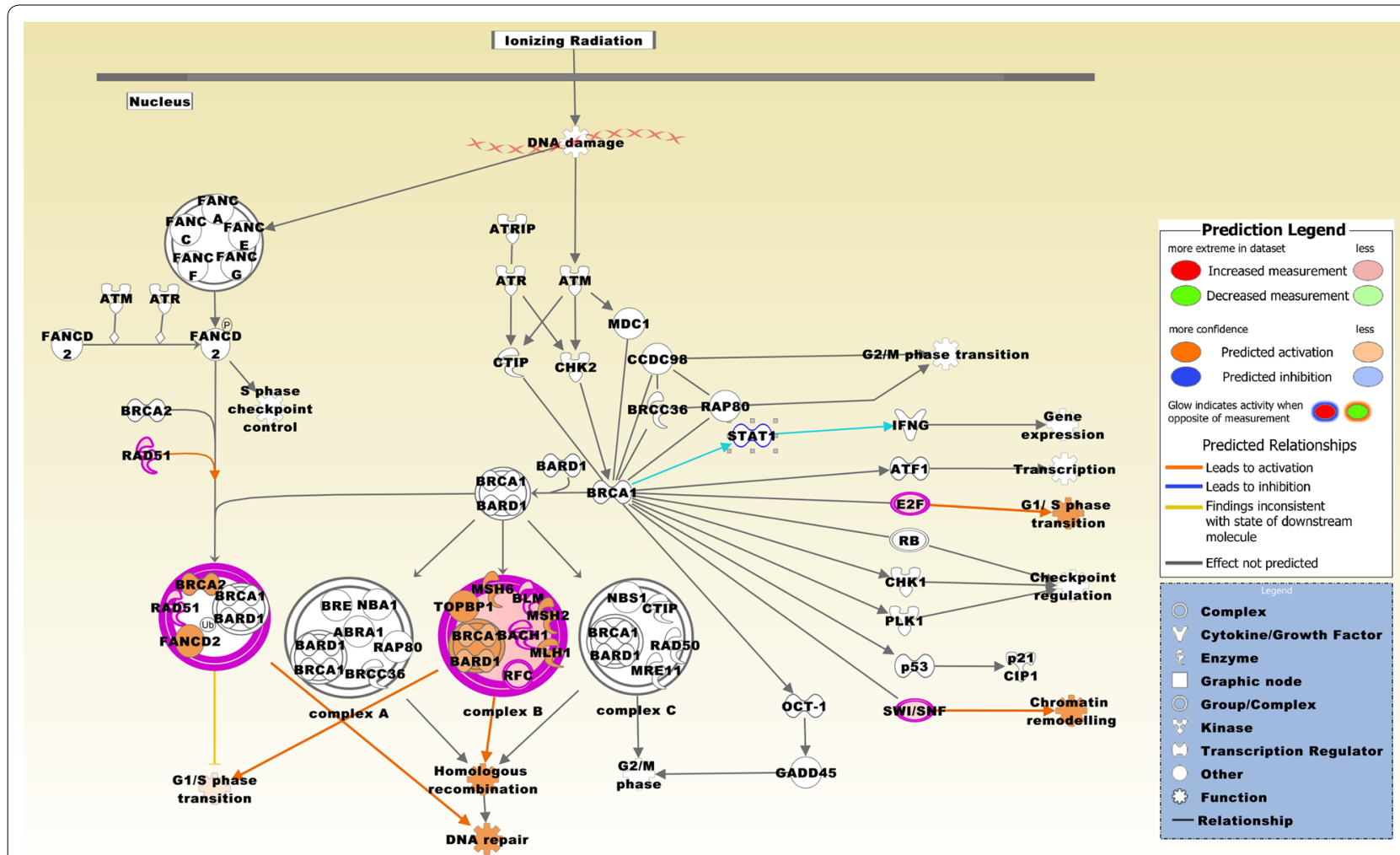

Fig. 5 The top canonical pathway is entitled, role of BRCA1 in DNA damage response. The pathway is based on the list of 370 differentially expressed probe sets and is displaying the expression values derived from the BCBM vs. BC comparison group (Additional file 1). Molecules that are comparably upregulated in BCBM include RAD51, DPF1, RFC4, E2F1, BRIP1, and BLM. The pathway was overlaid with the Molecule Activity Predictor to precalculate further molecular effects, as outlined in the prediction legend

three BCBM by gain, loss, or mosaic-loss. Amplification of ERBB2 was detected in Jed82_MT, sustaining IHC results. Mosaic-loss was revealed for the region comprising IDH1 in Jed81_MT and Jed82_MT whereas gain of $I D H 2$ was found in Jed89_MT. Conventional sequencing of $I D H 1$ and $I D H 2$ hotspot regions indicated no mutation (data not shown).

\section{Immunohistochemistry}

In all three BCBM, GFAP staining was revealed positive in interspersed astrocytes and in marginal gliosis areas but not in BCBM cells. ERBB2 staining was scored 1+ for Jed81_MT and Jed89_MT and 3+ for Jed82_MT (Fig. 11A-C). Nuclear staining for proliferation marker MKI67 was detected in $21 \pm 12.1 \%$ of Jed81_MT cells, in $45 \pm 14.7 \%$ of Jed $82 \_$MT cells, and in $39 \pm 7.8 \%$ of Jed89_MT cells (Fig. 11D-E).

\section{Whole exome sequencing}

Based on the chosen parameters, 615 mutations were reported in Jed81_MT, 544 in Jed82_MT, and 461 in Jed89_MT. Read depth for these mutations was on average 50.4 in Jed81_MT, 43.1 in Jed82_MT, and 51.3 in Jed89_MT. Taken together, the three most common mutation types, including those located in splice regions, were missense variants with $61 \%$, followed by frameshift variants with $17 \%$, and stop gained variants with $5 \%$. Mutation types with lower frequencies $(1 \%<$ range $<5 \%)$ included down- and upstream variants, splice region variants (others than listed under the most common types), inframe deletions, and inframe insertions. A comprehensive list of genes affected by mutations includes, e.g. BCL9L, CASP7, CDH1, ERBB4, FLT3, FOXD4, HEPACAM, LOXHD1, and PIK3C2G (Table 4). Conventional sequencing electropherograms 
Table 2 Top pathways and networks based on the differentially expressed probe sets

\begin{tabular}{|c|c|c|c|c|c|c|}
\hline \multirow[t]{2}{*}{ Category } & \multicolumn{3}{|c|}{$\begin{array}{l}\text { BCBM vs. } B C \text { intersecting } \\
\text { with } B C B M \text { vs. prBT }\end{array}$} & \multicolumn{3}{|c|}{ BCBM shared with prBT } \\
\hline & $p$-value & Overlap (\%) & Score & $p$-value & Overlap (\%) & Score \\
\hline \multicolumn{7}{|l|}{ Top canonical pathways } \\
\hline Role of BRCA1 in DNA damage response & $1.04 \mathrm{E}-03$ & 7.7 & & & & \\
\hline D-Myo-inositol $(1,4,5,6)$-tetrakisphosphate biosynthesis & 4.12E-03 & 5.1 & & & & \\
\hline D-Myo-inositol $(3,4,5,6)$-tetrakisphosphate biosynthesis & 4.12E-03 & 5.1 & & & & \\
\hline Estrogen-mediated S-phase entry & 5.13E-03 & 12.5 & & & & \\
\hline 3-phosphoinositide degradation & $6.98 \mathrm{E}-03$ & 4.7 & & & & \\
\hline EIF2 signaling & & & & $3.20 \mathrm{E}-04$ & 5.7 & \\
\hline Systemic lupus erythematosus signaling & & & & $6.10 \mathrm{E}-03$ & 4.8 & \\
\hline Oxidative phosphorylation & & & & 7.43E-03 & 6.1 & \\
\hline Superoxide radicals degradation & & & & 7.94E-03 & 25.0 & \\
\hline TNFR2 signaling & & & & $1.25 \mathrm{E}-02$ & 10.7 & \\
\hline \multicolumn{7}{|l|}{ Top networks related to diseases and functions } \\
\hline $\begin{array}{l}\text { Cell cycle, DNA replication, recombination, and repair, cellular assembly and } \\
\text { organization }\end{array}$ & & & 44 & & & \\
\hline Connective tissue disorders, developmental disorder, gastrointestinal disease & & & 39 & & & \\
\hline Cellular assembly and organization, inflammatory disease, inflammatory response & & & 37 & & & \\
\hline Cancer, cell death and survival, organismal injury and abnormalities & & & & & & 51 \\
\hline Hereditary disorder, neurological disease, organismal injury and abnormalities & & & & & & 48 \\
\hline Carbohydrate metabolism, drug metabolism, molecular transport & & & & & & 41 \\
\hline
\end{tabular}

are displayed for mutations in $L O X H D 1, E R B B 4$, and CASP7 (Fig. 12).

\section{Discussion}

The tumor biology of BCBM is complex and constitutes a challenge to identify new diagnostic and therapeutic targets that bear the capacity to enhance treatment efficiency of the disease. One of our major findings includes the upregulation of a number of snoRNAs compared to $\mathrm{BC}$ and prBT. This discovery is likely to be attributed to the fact that we employed whole transcript arrays on native preserved RNA derived from clinical specimens. Biostatistical tools and complementary molecular genetic techniques as high-density $\mathrm{CNV}$ analysis and WES allowed us to identify genes known to be related with $\mathrm{BC}$ and/or genes which may gain importance as new molecular biomarkers or targets.

\section{snoRNAs}

snoRNAs represent a conserved class of regulatory RNA molecules that are structurally and functionally categorized mainly into box C/D snoRNAs and box
H/ACA snoRNAs. They serve as a scaffold for assemblance of small nucleolar ribonucleoproteins in the nucleolus and are primarily involved in guiding posttranscriptional modification of ribosomal and snRNAs which is necessary for rRNA-controlled protein biogenesis [49-51]. Other functions include regulation of alternative splicing [52]. A number of snoRNAs associate with Cajal-body regions, which assemble to intra- and interchromosomal clusters. The majority of snoRNAs are mapping to introns of protein- and nonprotein coding genes. Intergenic human snoRNA promoters are enriched in E-boxes which are binding sites for MYC [53], and in $M Y C N$ amplified neuroblastomas, expression of several snoRNAs was reported to be higher compared to non-amplified neuroblastomas [54]. $M Y C N$ was in our BCBM significantly higher expressed than in $\mathrm{BC}$ and prBT. An in vitro and in vivo study demonstrated that overexpression of snoRNAs in $B C$ is critical for tumorigenicity and acts as a mechanism of nucleolar modulation of p53 for cancer cell survival [55]. Furthermore, RNA sequencing identified a number of snoRNAs that are associated with overall and/ 


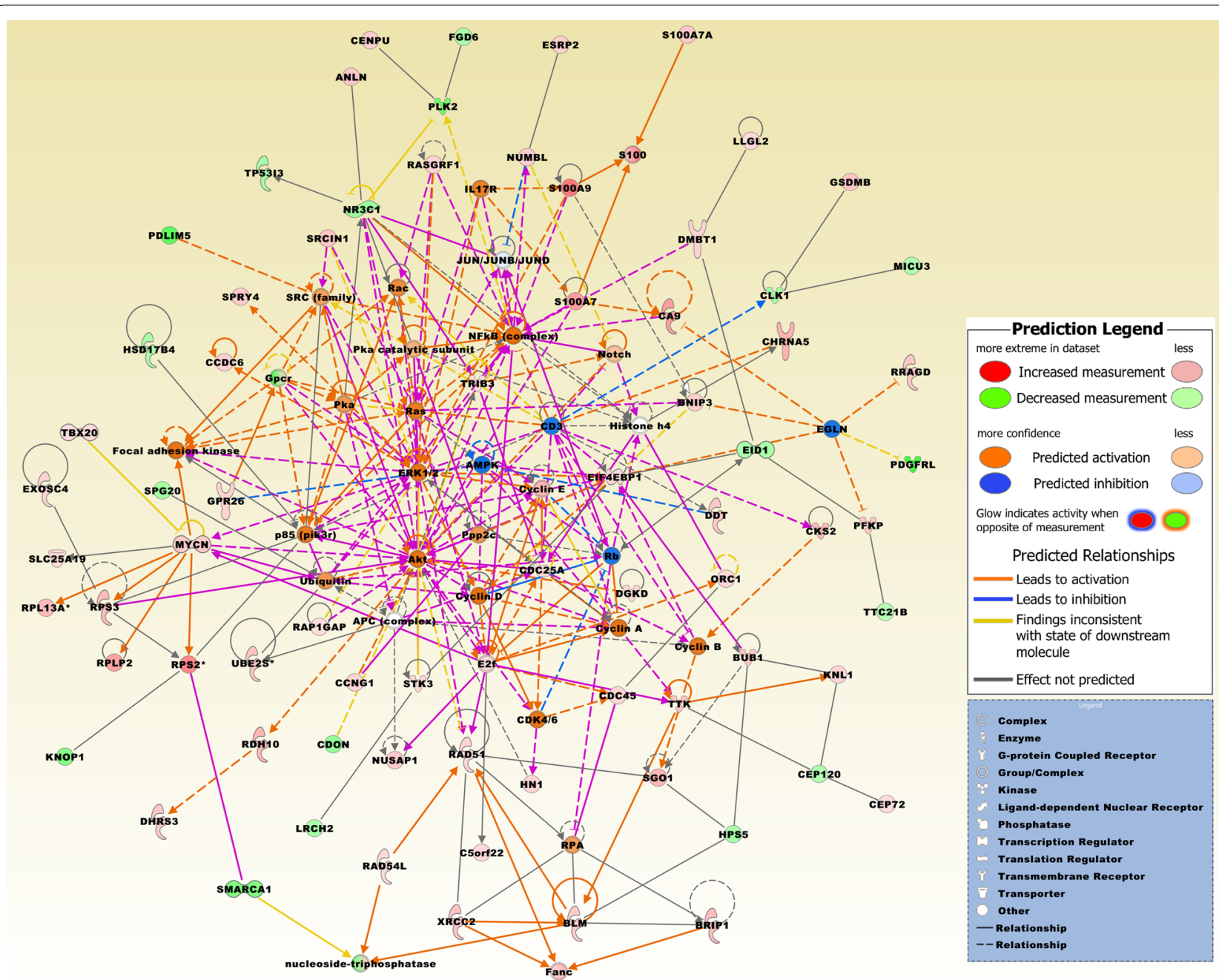

Fig. 6 The top three merged networks based on the 370 probe sets comprise molecules related to cell cycle, DNA replication, recombination, and repair, cellular assembly and organization, connective tissue and developmental disorders, gastrointestinal and inflammatory disease and inflammatory response (Table 2). Expression values are derived from the comparison group BCBM vs. BC (Additional file 1). Upregulated molecules include ANLN, BLM, BNIP3, BRIP1, BUB1, C5orf22, CA9, CCDC6, CDC25A, CDC45, CENPU, CEP72, CHRNA5, CKS2, DDT, DGKD, DMBT1, E2f, E2F1, EIF4EBP1 (alias 4EBP1), ESRP2, EXOSC4, GPR26, GSDMB, HN1, LLGL2, KNL1 (CASC5), MYCN, NUSAP1, ORC1, PFKP, RAD51, RAD54L, RAP1GAP, RASGRF1, RDH10, RRAGD, RPL13A (SNORD34, SNORD35A), RPLP2 (SNORA52), RPS2 (SNORA10), RSP3 (SNORD15A), S100A7, S100A7A, S100A9, SGO1 (SGOL1), SLC25A19, SPRY4, SRCIN1, STK3, TBX20, TRIB3, TTK, UBE2S, and XRCC2. Downregulated molecules include CCNG1, CDON, CEP120, CLK1, DHRS3, EID1, FGD6, HPS5, HSD17B4, KNOP1, LRCH2, MICU3, NR3C1, NUMBL, PDGFRL, PDLIM5, PLK2, SMARCA1, SPG20, TP5313, and TTC21B. Integrative network molecules comprise Akt, AMPK, APC (complex), CD3, CDK4/6, Cyclin A, Cyclin B, Cyclin D, Cyclin E, E2f, EGLN, ERK1/2, Fanc, Focal adhesion kinase (FAC), Gpcr, Histone h4, IL17R, JUN/JUNB/JUND, NFkB (complex), Notch, nucleoside-triphosphatase, Ppp2c, Rb, RPA, S100, and Ubiquitin. Of notice, key nodes as Akt, ERK1/2, NFkB, and Ras were in a predicted activation stage. Asterisks mark molecules with more than one probe set. The pathway was overlaid with the Molecule Activity Predictor to precalculate further molecular effects, as outlined in the prediction legend

or relapse-free survival in $\mathrm{BC}$ [56]. Regulative functions have been described for a number of snoRNAs from our 370 probe sets. For example, among other genes, SNORD34, SNORD35A, SNORA38B, and SNORA71A were comparably downregulated in a glioblastoma multiforme (GBM) cell line where expression of the developmental gene HOXA10 was silenced by siRNA treatment leading to a signal cascade that impaired the homologous recombination pathway and ultimately diminished temozolomide resistance [57]. Furthermore, 


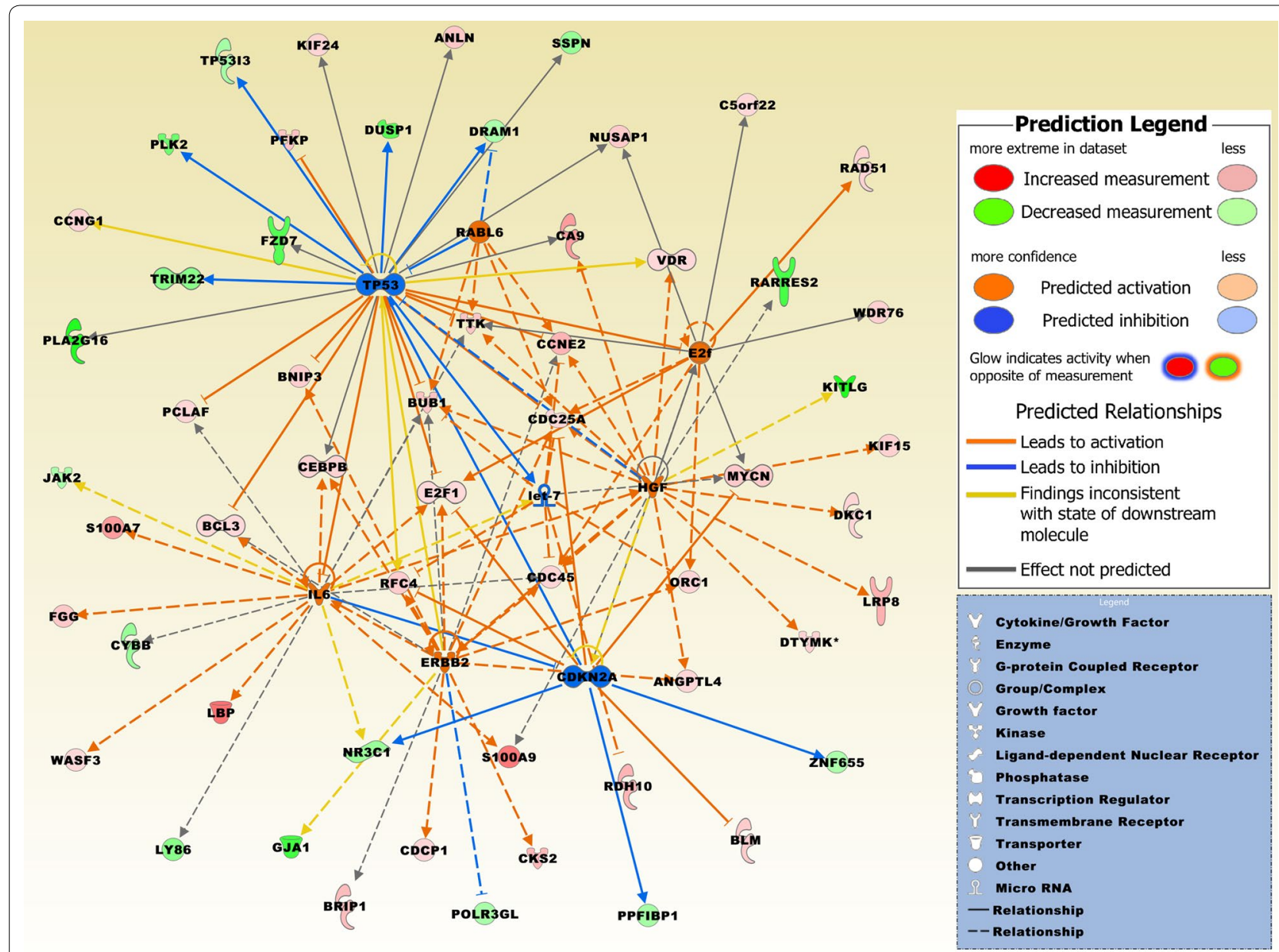

Fig. 7 A merged network based on eight upstream regulators, of which E2f, ERBB2, IL6, HGF, and RABL6 were in a predicted activation state ( $z$-score $>2$ ) and let-7, TP53, and CDKN2A were in a predicted inhibition state (z-score $<-2)$. P-value of overlap for all upstream regulators is $<0.0029$. The network is based on the list of 370 differentially expressed probe sets and displays the expression values derived from the comparison group BCBM vs. prBT (Additional file 1). Upregulated molecules include ANGPTL4, ANLN, BCL3, BLM, BNIP3, BRIP1, BUB1, C5orf22, CA9, CCNE2, CCNG1, CDC25A, CDC45, CDCP1, CEBPB, CKS2, DKC1, DTYMK, E2F1, FGG, KIF15, KIF24, LBP, LRP8, MYCN, NUSAP1, ORC1, PCLAF, PFKP, RAD51, RDH10, RFC4, S100A7, S100A9, TTK, VDR, WASF3, and WDR76. Downregulated molecules include CYBB, DRAM1, DUSP1, FZD7, GJA1, JAK2, KITLG, LY86, NR3C1, PLAZG16, PLK2, POLR3GL, PPF1BP1, RARRES2, SSPN, TRIM22, and ZNF655. Asterisk marks molecule with more than one probe set. The pathway was overlaid with the Molecule Activity Predictor to precalculate further molecular effects, as outlined in the prediction legend

SNORA71A and SNORA71C were two of the top upregulated snoRNAs upon retinoic acid and/or thalidomide treatment of GBM [58]. SNORD15A and SNORA38 are known to be involved in regulation of chromatin structure [59]. In MCF7 BC cells, SNORD34 was one of two snoRNAs that was found to bind to the nucleic acid-binding protein $\mathrm{YB}-1$ which itself is associated with unfavorable prognosis of $\mathrm{BC}$ patients [60]. Among a number of differentially expressed genes, SNORA2A was identified as a highly expressed transcript in, nonsentinel lymph node positive, sentinel lymph nodes metastasis [61]. These data indicate that the set of snoRNAs which were all upregulated in our BCBM represent new biomarkers with a feasibility to gain diagnostic and therapeutic relevance. Important in the clinical context, as snoRNAs are detectable in blood serum and 


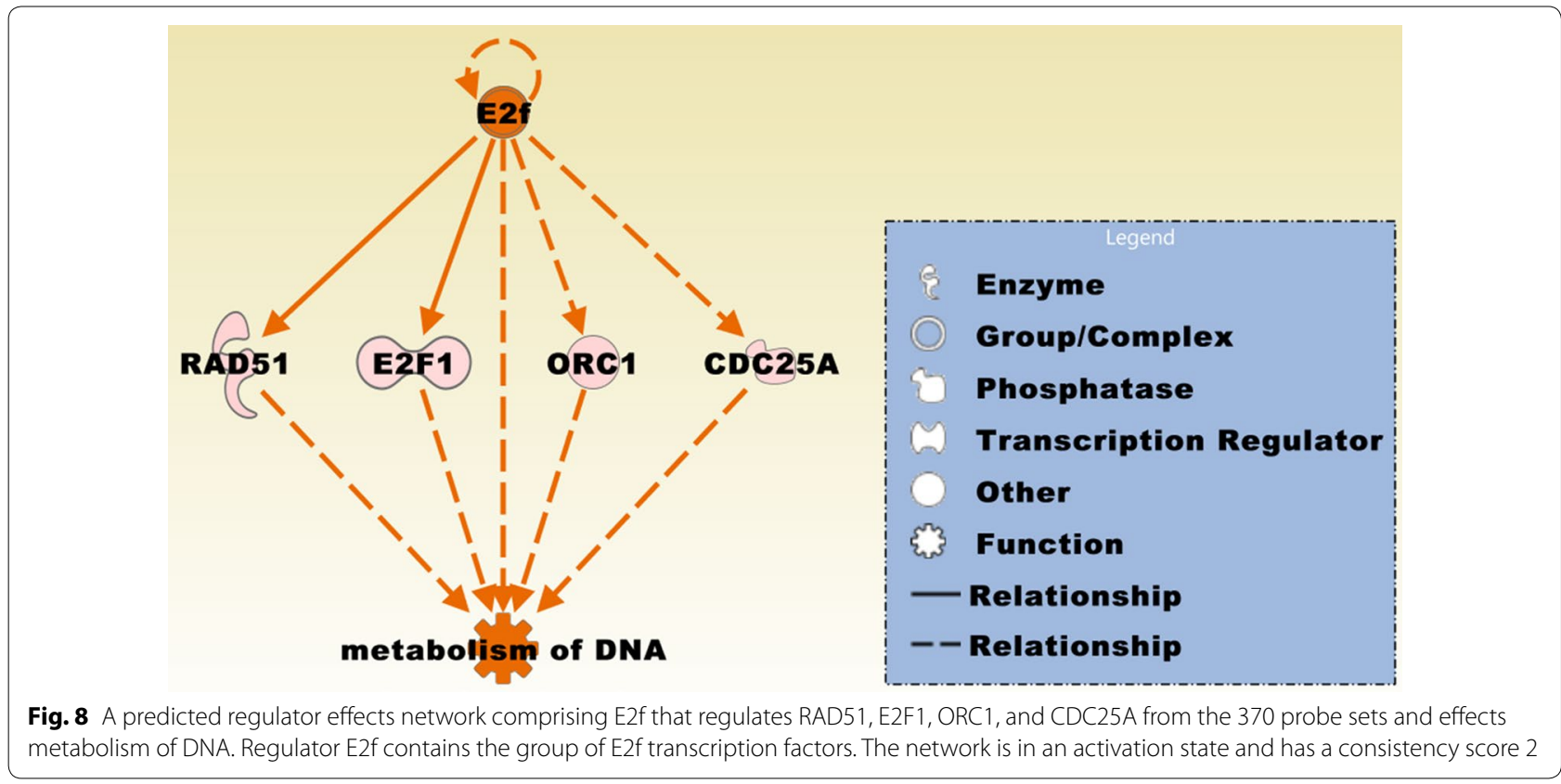

plasma, quantitative and qualitative detection of snoRNAs in BC patients could become a diagnostic tool to identify patients at risk for brain metastasis [62]. The small Cajal body-specific RNA SCARNA22 which maps to the intron of the Wolf-Hirschhorn syndrome candidate 1 (WHSC1) gene is known to be involved in cell proliferation and stress response in a subset of multiple myelomas [63]. Besides snoRNAs, a number of snRNAs were comparably upregulated in BCBM which could be attributed to impairment of TP53 mediated repression of snRNA transcription by polymerases II and III [64].

\section{$\mathrm{BC}$ associated genes}

BRCA1 exerts critical functions in S-phase activation and DNA damage repair [65]. The BRCA1 canonical pathway genes BRIP1, BLM, DPF1, E2F1, RAD51, and RFC4 were in our study comparably higher expressed in BCBM. Recent RNA and protein expression studies revealed a higher RAD51 expression in BCBM compared to matched primary BC $[66,67]$. Furthermore, in transplantation mouse models, RAD51 competent primary tumors metastasized to bone and brain, whereas primary tumors depleted of RAD51 showed an inhibition of metastatic seedings [66]. Overexpression of RAD51 brain metastatic cells may be a response mechanism to genotoxic stress caused by neuro-inflammatory microenvironment of the brain [67]. In an experimental model system, cancer stem cells in BRCA1 mutant TNBC which were resistant to PARP inhibition showed higher RAD51 expression [68]. RAD51 downregulation by shRNA resulted in growth inhibition of TNBC leading to the suggestion that RAD51 silencing may increase the efficiency of PARP inhibitors. Higher expression of the BRCA interacting protein BRIP1 was detected in ER and PR negative, ERBB2 positive $B C$ and was associated with unfavourable prognosis [69]. A bioinformatics analysis revealed that DPF1, which is a member of the neuron-specific chromatin remodeling complex, is among the most consistently overexpressed genes in various cancers analysed by the TCGA consortium [70]. In MCF7 BC cells, E2F1 downregulation by the sesquiterpene lactone artemisinin resulted in cell cycle arrest whereas constitutive E2F1 expression reversed the antiproliferative effect showing the critical function of E2F1 in promoting BC growth [71]. RFC4 and $B L M$ are known to be upregulated in $B R C A 1$ mutant $\mathrm{BC}$ [72]. A $B R C A 1$ deficient-like signature has been identified in ERBB2 positive $B C B M$ compared to non-brain metastatic $\mathrm{BC}$ [33]. Acquired $E R B B 2$ alterations leading to elevated ERBB2 expression are known to be frequently associated with BCBM [73]. A MYC driven accumulation of the oncometabolite 2-hydroxyglutarate has been found in $\mathrm{BC}$, primarily in ER negative and basal-like types [74, 75]. In our 370 probe sets, the mitochondrial $I D H 2$ and $M Y C N$ were upregulated in BCBM.

\section{Whole exome sequencing}

In vitro and in vivo experiments demonstrated that mutant BCL9L impairs CASP2 function in aneuploid colorectal cancer cells resulting in tolerating chromosome missegregation, independently of the functional TP53 status [76]. Missense mutations at codon 400 in $C D H 1$ and at codon 570 in $C D H 10$ have been 


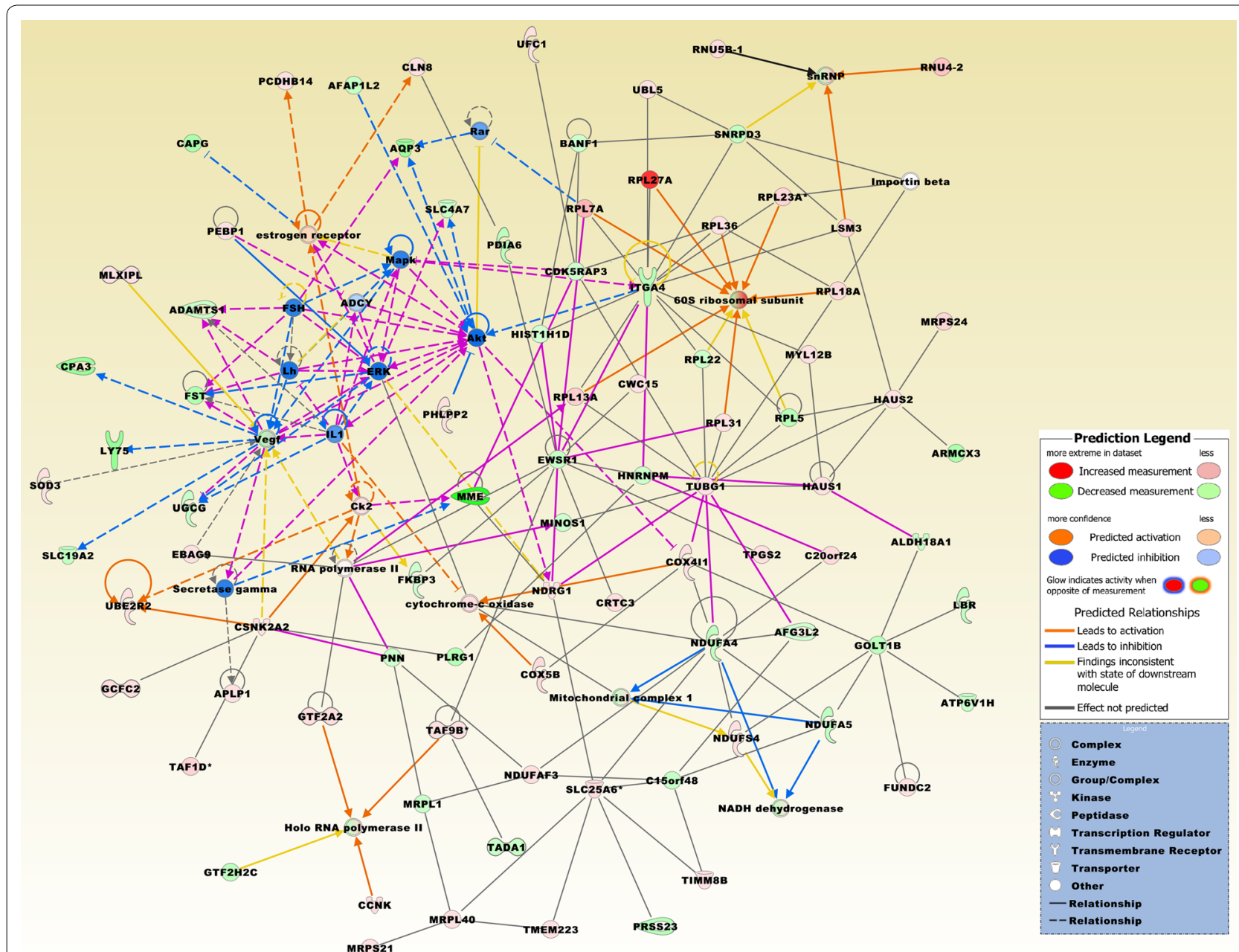

Fig. 9 The top three merged networks based on the 643 probe sets that were common between BCBM and prBT but significantly differentially expressed between BCBM and BC include molecules that are related to cancer, cell death and survival, organismal injury and abnormalities, hereditary disorder, neurological disease, carbohydrate and drug metabolism, and molecular transport. Molecules that are upregulated in BCBM compared to BC include, APLP1, C20orf24, CCNK, CLN8, COX411, COX5B, CRTC3, CSNK2A2, CWC15, EBAG9, FUNDC2, GCFC2, GTF2A2, HAUS1, HAUS2, LSM3, MLXIPL, MRPL40, MRPS21, MYL12B, NDRG1, NDUFAF3, NDUFS4, MRPS24, PCDHB14, PEBP1, PHLPP2, RNU4-2, RNU5B-1, RPL7A (SNORD36B), RPL13A (SNORD32A), RPL18A (SNORA68), RPL23A (SNORD42A), RPL27A (SNORA45A), RPL31, RPL36, SOD3, TAF1D, TAF9B, TIMM8B, TMEM223, TPGS2, TUBG1, UBE2R2, UBL5, UFC1. Downregulated molecules include ADAMTS1, AFAP1L2, AFG3L2, ALDH18A1, ARMCX3, AQP3, ATP6V1H, C15orf48, CAPG, CDK5RAP3, CPA3, EWSR1, FKBP3, FST, GOLT1B, GTF2H2C (paralogue GTF2H2C_2 in data set), HIST1H1D, HNRNPM, ITGA4, LBR, LY75 (LY75CD302 readthrough in data set), MINOS1, MME, MRPL1, NDUFA4, NDUFA5, PDIA6, PLRG1, PNN, PRSS23, RPL5 (SNORD21), RPL22, SLC19A2, SLC25A6, SLC4A7, SNRPD3, TADA1, and UGCG. Integrative molecules comprise 60S ribosomal subunit, ADCY, Akt, Ck2, cytochrome-c oxidase, ERK, estrogen receptor, FSH, IL1, Importin beta, Lh, Mapk, mitochondrial complex 1, NADH dehydrogenase, Rar, Holo RNA polymerase II, RNA polymerase II, secretase gamma, snRNP, and Vegf. Asterisks mark molecules with more than one probe set. The pathway was overlaid with the Molecule Activity Predictor to precalculate further molecular effects, as outlined in the prediction legend

previously reported in gastric carcinoma (COSMIC IDs COSM1159626, COSM20771) and urinary tract carcinoma (COSM1311079), respectively [47]. Women with a constitutional $C D H 1$ mutation are at elevated risk for breast cancer [77]. In gastric cancer cells, CYFIP2 silencing resulted in enhanced proliferation and colony formation, decreased apoptosis and induced resistance to 5-FU [78]. E2F7 is a repressor of transcription by interacting with co-repressor CtBP2 and transcription factor E2F1 which is an activator of the G1/S phase [79]. DAPK1 is known as a critical regulator of apoptosis and autophagy [80]. The missense mutation in FLT3 has been earlier detected in a colon carcinoma and an endometrioid carcinoma (COSM946463). FOXD4 has been described as an essential factor for neuronal differentiation [81]. The frameshift mutation in FOXD4L1 leading to a truncated 

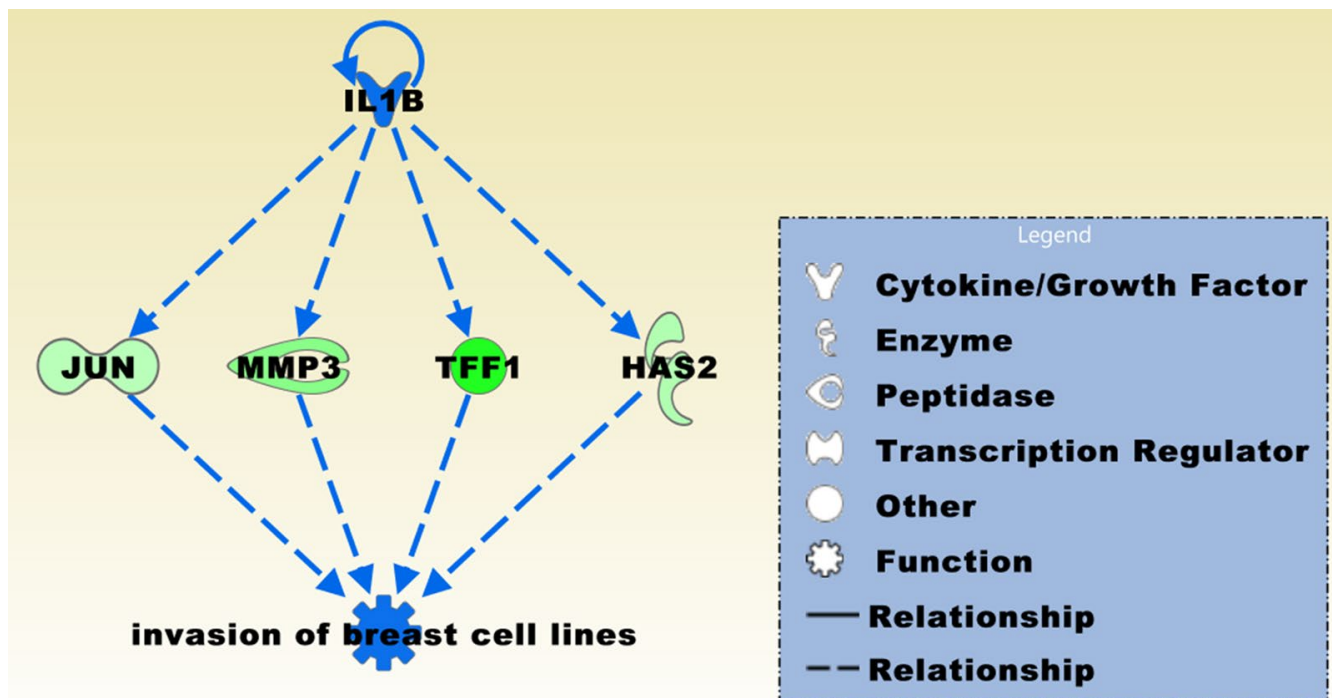

Fig. 10 A predicted regulator effects network comprising IL1B that regulates JUN, MMP3, TFF1, and HAS2 from the 643 probe sets and effects invasion of breast cell lines. The network is in an inhibition state and has a consistency score 2

Table 3 CNVs in TP53, BRCA1, BRCA2, ERBB2, IDH1, and IDH2

\begin{tabular}{|c|c|c|c|c|}
\hline Case & Copy number state & No. of OMIM genes ${ }^{1}$ & Important cancer genes & Array nomenclature ${ }^{2}$ \\
\hline Jed81_MT & Mosaic-loss & 226 & TP53 & arr[hg19] 17p13.3p11.2(525-20,011,122)x1-2 \\
\hline Jed82_MT & Mosaic-loss & 291 & TP53 & $\operatorname{arr}[$ hg 19] 17p13.3q12(525-32,273,420)x1-2 \\
\hline Jed89_MT & Mosaic-loss & 289 & TP53 & arr[hg19] 17p13.3q11.2(525-31,245,235)×1-2 \\
\hline Jed82_MT & Loss & 6 & $B R C A 1$ & arr[hg19] 17q21.31(41,251,930-41,829,105)x1 \\
\hline Jed81_MT & Gain & 5 & $B R C A 2$ & arr[hg19] 13q13.1q13.2(32,960,791-34,576,864)×3 \\
\hline Jed89_MT & Mosaic-loss & 103 & $B R C A 2$ & arr[hg19] 13q11q14.2(19,436,286-47,693,486)×1-2 \\
\hline Jed82_MT & Gain & 23 & ERBB2 & $\operatorname{arr}[$ hg19] 17q12q21.1 $(37,063,504-38,179,492) \times 4$ \\
\hline Jed81_MT & Mosaic-loss & 435 & IDH1 & arr[hg19] 2q14.3q37.3(123,839,696-242,275,944)×1-2 \\
\hline Jed82_MT & Mosaic-loss & 75 & IDH1 & $\operatorname{arr}[\mathrm{hg} 19] 2 \mathrm{2q32.3q35}(192,641,695-216,741,600) \times 1-2$ \\
\hline Jed89_MT & Gain & 11 & $\mathrm{IDH} 2$ & arr[hg19] 15q26.1 (90,592,464-91,543,761)x4 \\
\hline
\end{tabular}

${ }_{1}^{1}$ OMIM, online mendelian inheritance in man; $^{2}$ on average $91 \%$ overlap of affected regions between duplicate assays

variant is affecting its C-terminal repressor function [82]. In non- small cell lung carcinoma, knockdown of HEPACAM expression stimulated cell proliferation, migration, and metastasis [83]. The stop gained mutation in HEPACAM identified in Jed89_MT has been previously reported in a breast tumor sample (COSM240098). Same like, the detected LOXHD1 missense mutation in Jed81_MT has been previously detected in an invasive breast carcinoma (COSM1480342). Expression of PIK3C2G is mainly limited to breast, liver, and prostate and its functions remains to be elucidated [84]. Some of the identified mutations, as those that were recurrently observed, may represent rare constitutional variants, yet may contribute to the genetic burden of BCBM.

\section{Comparison of expression data to $\mathrm{BC}$ with $\mathrm{BCBM}$}

To assess in how far expression profiles between our 370 probe sets are shared with those from $\mathrm{BC}$ with a known BCBM, we substituted in an ANOVA the three BCBM from our two comparison groups, $B C B M$ vs. $B C$ and BCBM vs. prBT, with three TNBC for which a brain metastatic process has been reported (Dr. S. Ambs et al., pers. comm., Feb., 2017). These three samples (GSM927034, GSM927039, and GSM927040) were derived from GEO submission GSE37751 [75]. ANOVA generated 501 probe sets of which 49 intersected with our 370 probe sets. The 49 probe sets comprise, besides a number of snoRNAs and other non-coding RNAs, several annotated coding genes including ANGPTL4, BCL3, CETN3, EID1, 


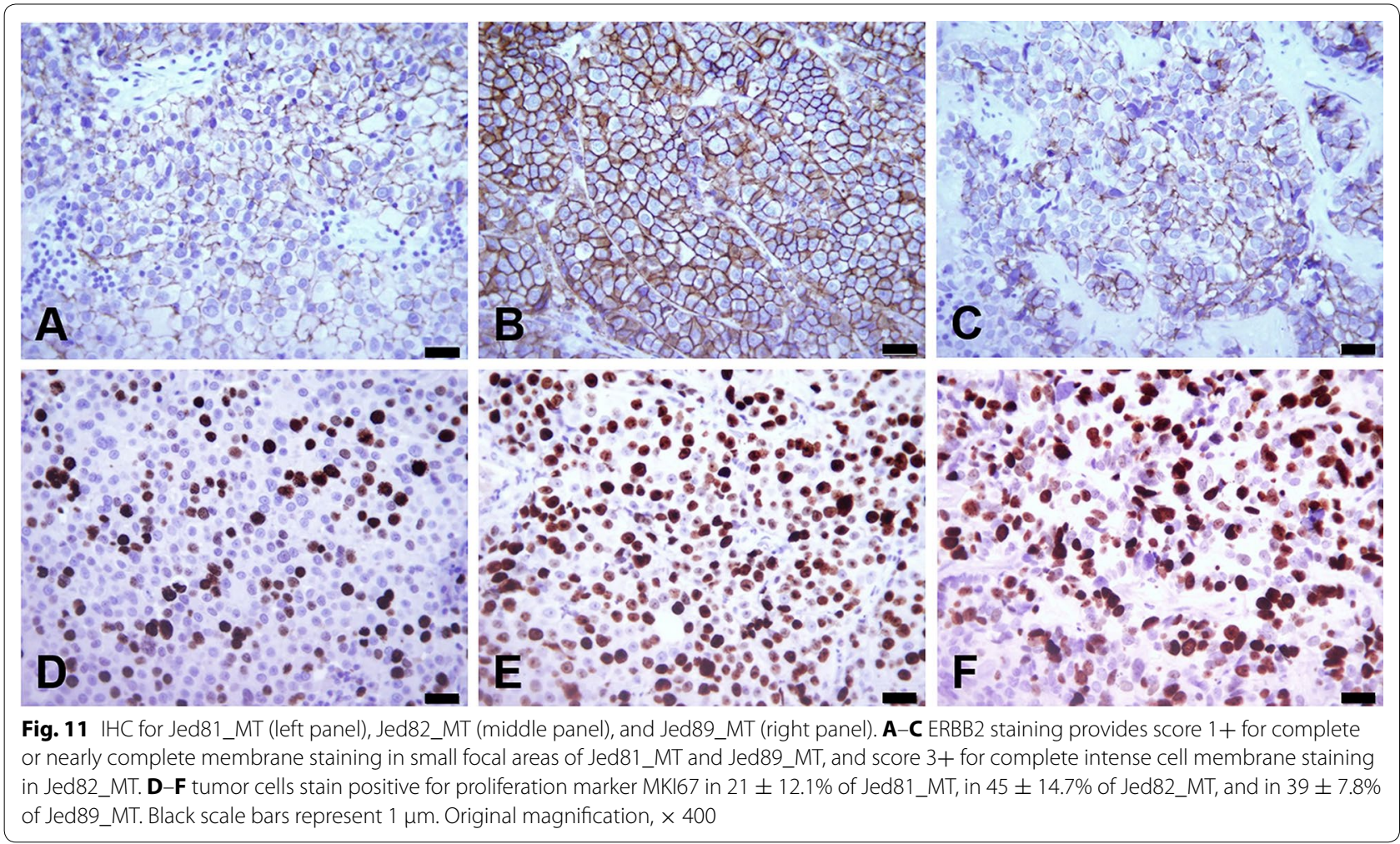

GABARAPL1, GRHL2, HPS5, KLHDC2, PLA2G16, PLK2, SERPINB1, SESN3, TMX4, TMEM99, TRIM22, UBAP2L, $U C P 2$, and $W W C 2$. With the exception of GRHL2, which was comparably downregulated in the 49 probe sets but upregulated in the 370 probe sets, all other intersecting genes were either up- or downregulated in the same direction. ANGPTL4 is a TGF $\beta$ target gene in cancer cells and in vitro and in vivo experiments demonstrated that it impairs integrity of vascular endothelial cell layers fostering $\mathrm{BC}$ cell passage during lung metastatic process [85]. In mice models, knockdown of Bcl3 in ErbB2 positive $B C$ resulted in decreased cell motility and metastatic progression without affecting primary tumor growth; however, resulted in severe reduction of lung metastatic tumors [86]. Functional downregulation of GRHL2 is known to be associated with elevated cell proliferation; yet, its protein expression has been found to be downregulated at the invasion front of primary breast tumors [14]. GABARAPL1 is known to be involved in autophagy and its higher expression was associated with favorable prognosis in BC with lymph node metastases [87]. Based on its cell cycle regulator function PLK2 is known as a tumor suppressor [88]. TMX4 is a thioredoxin-related molecule that is localized to the ER and involved in protein folding. UBAP2L is a critical factor for hematopoietic stem cell activity and exhibits critical functions in glioma cell growth $[89,90]$. In BC cell lines, $U C P 2$ silencing in combination with cytotoxic treatment led to decrease in cell viability and increase in ROS production, apoptosis, and autophagy [91]. Furthermore, higher UCP2 expression in $\mathrm{BC}$ patients corresponded with unfavorable prognosis.

\section{Conclusions}

Using complementary state-of-the-art techniques this study provides a comprehensive overview of the complex tumor biology of BCBM reflecting the challenge to identify effective molecular biomarkers with clinical implications, especially in view that targeted therapy options for $\mathrm{BCBM}$ are limited. Among new findings with the capacity to gain clinical relevance is the detection of overexpressed snoRNAs, which represented more than $5 \%$ of the 370 probe sets that differentiate $\mathrm{BCBM}$ from $\mathrm{BC}$ and 
Table 4 Selection of gene mutations identified by WES

\begin{tabular}{|c|c|c|c|c|c|c|c|}
\hline Jed81_MT ${ }^{1,2}$ & Jed82_MT ${ }^{1,2}$ & Jed89_MT ${ }^{1,2}$ & Gene & Read depth & Consequence & HGVSc $^{3}$ & HGVSp $^{4}$ \\
\hline he & & & ANLN & 11 & Inframe insertion & $\begin{array}{l}\text { NM_018685.2:c.881_884de } \\
\text { ICTTCinsCTTCTTC }\end{array}$ & $\begin{array}{c}\text { NM_018685.2:C.881_884 } \\
\text { delCTTCinsCTTCTTC }\end{array}$ \\
\hline \multirow[t]{2}{*}{ he } & & & $B C L 9 L$ & 19 & Missense & NM_182557.2:C.1276G > C & $\begin{array}{l}\text { NP_872363.1:p. } \\
\text { Glu426Gln }\end{array}$ \\
\hline & he & ho & CASP7 & 18 & Frameshift & $\begin{array}{l}\text { NM_001267057.1:c.128_13 } \\
\text { OdelTTTinsT }\end{array}$ & $\begin{array}{l}\text { NP_001253986.1:p. } \\
\text { Leu44SerfsTer70 }\end{array}$ \\
\hline \multirow[t]{3}{*}{ he } & & & $\mathrm{CDH} 1$ & 26 & Missense & NM_004360.3:c.1198G >C & $\begin{array}{l}\text { NP_004351.1:p. } \\
\text { Asp400His }\end{array}$ \\
\hline & & he & $\mathrm{CDH} 9$ & 33 & Missense & NM_016279.3:c.643G > T & $\begin{array}{l}\text { NP_057363.3:p.Gly- } \\
\text { 215Cys }\end{array}$ \\
\hline & he & & $\mathrm{CDH} 10$ & 128 & Missense & NM_006727.3:c.1708G > C & $\begin{array}{l}\text { NP_006718.2:p. } \\
\text { Asp570His }\end{array}$ \\
\hline ho & ho & ho & CYFIP2 & $12,26,16$ & Frameshift & $\begin{array}{l}\text { NM_001037333.1:c.280_28 } \\
\text { 1delCCinsCCC }\end{array}$ & $\begin{array}{l}\text { NP_001032410.1:p. } \\
\text { Gln95ProfsTer15 }\end{array}$ \\
\hline \multirow[t]{10}{*}{ he } & & & DAPK1 & 19 & Missense & NM_004938.2:c.3090G > T & $\begin{array}{l}\text { NP_004929.2:p. } \\
\text { Gln1030His }\end{array}$ \\
\hline & he & & E2F7 & 16 & Missense & NM_203394.2:C.1292C > T & $\begin{array}{l}\text { NP_976328.2:p.Pro- } \\
\text { 431Leu }\end{array}$ \\
\hline & he & & ERRB 4 & 24 & Missense & NM_005235.2:c.257A > T & NP_005226.1:p.Tyr86Phe \\
\hline & & he & ERRB 4 & 31 & Missense & NM_005235.2:c.2621A > T & $\begin{array}{l}\text { NP_005226.1:p.Glu- } \\
\text { 874Val }\end{array}$ \\
\hline & he & & FLT3 & 14 & Missense & NM_004119.2:C.2957C > T & $\begin{array}{l}\text { NP_004110.2:p.Pro- } \\
\text { 986Leu }\end{array}$ \\
\hline & & ho & FOXD4 & 50 & Frameshift & $\begin{array}{l}\text { NM_207305.4:C.1264_12 } \\
\text { 71delGTTTTTTTinsGTT } \\
\text { TTTTTT }\end{array}$ & $\begin{array}{l}\text { NP_997188.2:p. } \\
\text { Leu424PhefsTer59 }\end{array}$ \\
\hline & & ho & FOXD4 & 13 & Frameshift & $\begin{array}{l}\text { NM_207305.4:c.755_756de } \\
\text { IGGinsGGG }\end{array}$ & $\begin{array}{l}\text { NP_997188.2:p.Arg- } \\
\text { 253GlufsTer230 }\end{array}$ \\
\hline & & he & FOXD4L 1 & 74 & Frameshift & $\begin{array}{l}\text { NM_012184.4:c.763_764de } \\
\text { IGGinsGGG }\end{array}$ & $\begin{array}{l}\text { NP_036316.1:p.Arg- } \\
\text { 256GlufsTer72 }\end{array}$ \\
\hline & & ho & HEPACAM & 24 & Stop gained & NM_152722.4:C.298C > T & $\begin{array}{l}\text { NP_689935.2:p. } \\
\text { Arg100Ter }\end{array}$ \\
\hline & he & & IDH3G & 15 & Frameshift & $\begin{array}{l}\text { NM_004135.3:c.562_563d } \\
\text { elAGinsA }\end{array}$ & $\begin{array}{l}\text { NP_004126.1:p. } \\
\text { Ser188ThrfsTer2 }\end{array}$ \\
\hline he & & & LOXHD1 & 27 & Missense & NM_144612.6:C.3124G > A & $\begin{array}{l}\text { NP_653213.6:p. } \\
\text { Val1042lle }\end{array}$ \\
\hline he & & & MAP4K3 & 29 & $\begin{array}{l}\text { Stop gained, splice } \\
\text { region }\end{array}$ & NM_003618.3:c.2539G > T & $\begin{array}{l}\text { NP_003609.2:p.Glu- } \\
\text { 847Ter }\end{array}$ \\
\hline ho & ho & & MMP12 & 27,19 & Frameshift & $\begin{array}{l}\text { NM_002426.4:C.630_631de } \\
\text { ICAinsAAA }\end{array}$ & $\begin{array}{l}\text { NP_002417.2:p. } \\
\text { Thr211AsnfsTer261 }\end{array}$ \\
\hline \multirow[t]{4}{*}{ he } & & he & PIK3C2G & 24,14 & Inframe deletion & $\begin{array}{l}\text { NM_004570.4:C.384_387de } \\
\text { ICCCCinsC }\end{array}$ & $\begin{array}{l}\text { NP_004561.3:p. } \\
\text { Pro129del }\end{array}$ \\
\hline & & he & PPARG & 38 & Missense & NM_015869.4:C.1360C > A & $\begin{array}{l}\text { NP_056953.2:p.Pro- } \\
\text { 454Thr }\end{array}$ \\
\hline & & he & RAD54B & 131 & Frameshift & $\begin{array}{l}\text { NM_012415.3:c.2732_2733 } \\
\text { delAGinsA }\end{array}$ & $\begin{array}{l}\text { NP_036547.1:p.Ter- } \\
\text { 911TyrfsTer16 }\end{array}$ \\
\hline & & he & RASGRP3 & 20 & Missense & NM_170672.2:C.492G > T & $\begin{array}{l}\text { NP_733772.1:p.Glu- } \\
\text { 164Asp }\end{array}$ \\
\hline
\end{tabular}

\footnotetext{
${ }^{1}$ he, heterozygous mutation; ${ }^{2}$ ho, homozygous mutation; ${ }^{3} \mathrm{HGVSc}$, Human Genome Variation Society notation in the cDNA; ${ }^{4}$ HGVSp, Human Genome Variation
} Society notation in the protein 

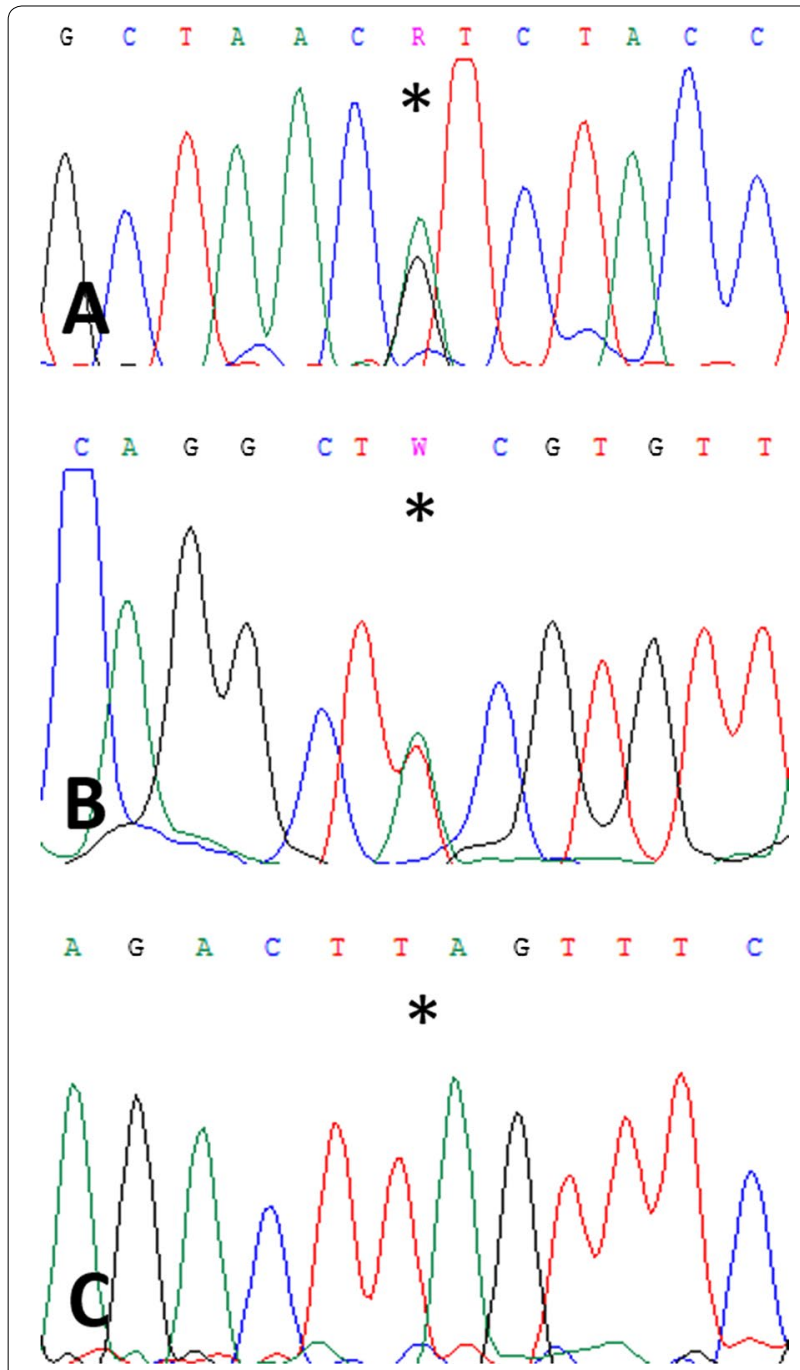

Fig. 12 Mutations identified by WES and verified by conventional sequencing. A LOXHD1 missense mutation c.3124G > A; p.Val1042lle in Jed81_MT. B ERBB4 missense mutation c.257A > T; p.Tyr86Phe in Jed82_MT. C CASP7 frameshift (fs) mutation C.128_130delTTTinsT; p.Leu44SerfsTer70 in Jed89_MT. Asterisks mark missense and frameshift/homozygous deletion mutations, respectively. Sequence reactions from the reverse strand are shown in sense strand view

prBT. The specificity of the probe sets was further demonstrated in the biofunctional analysis showing, e.g. that top merged networks have key nodes as Akt, ERK1/2, $\mathrm{NFkB}$, and Ras in the predicted activation stage. Furthermore, downregulation of four $\mathrm{BC}$ cell line invasion markers in a data set that was shared between BCBM and prBT implies reprogramming of the invasive behavior of the BCBM. A number of cancer associated genes were involved in CNVs including mosaic-losses and numerous mutations, some of which in known $\mathrm{BC}$ associated genes, were detected by WES.

\section{Additional files}

Additional file 1. Probe sets differentially expressed in breast cancer
brain metastases (BCBM) compared to breast cancer (BC) and primary brain tumors (prBT).

Additional file 2. Exon expression levels for a number of cancer associated genes. A, BRCA1; B, BRCA2; C, ERBB2; D, TP53; E, ER1 (ESR1); F, PR (PGR);

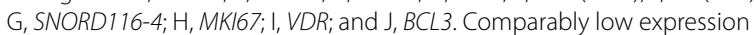
of a number of exon probes can be presumbably attributed to splicing events of transcripts; asterisks mark examples. $B C L 3$ is upregulated on the gene expression level (Additional file 1). Notably, 5 ' located exons of ERBB2 are lower expressed than $3^{\prime}$ located exons in PrBT and expression levels of the two probes covering the SNORD116-4 transcript diverge in BCBM and prBT compared to BC. Exon probes are displayed from $5{ }^{\circ}$ (left) to $3^{\prime}$ (right) of the transcripts. Blue and red colors in heat maps refer to lower and higher expression, respectively.

\section{Abbreviations}

ANOVA: analysis of variance; BBB: blood brain barrier; $B C$ : breast cancer; $B C B M$ : breast cancer brain metastasis; $\mathrm{CNV}$ : copy number variation; FC: fold change; FDR: false discovery rate; GO: gene ontology; GBM: glioblastoma multiforme; IHC: immunohistochemistry; PCA: principal component analysis; prBT: primary brain tumors; snoRNA: small nucleolar RNA; snRNAs: small nuclear RNAs; TNBC: triple negative $B C ; W E S$ : whole exome sequencing.

\section{Authors' contributions}

Conceived and designed the experiments: HJS, MB, SK, AD, DH, AM, FKA, JM, AJ, FG, HC, SSB, AGC, and MHA. Performed the experiments: HJS, SK, AD, DH, $J M, A G$, and HC. Analyzed the data: HJS, MB, SK, AD, DH, FKA, JM, FG HC, and SSB. Contributed reagents, materials and analysis tools: HJS, MB, SK, AD, DH, AM, FKA, AJ, HC, AGC, and MHA. Wrote and edited the paper: HJS, DH, SSB, AGC, and MHA. All authors read and approved the final manuscript.

\section{Author details}

${ }^{1}$ Center of Excellence in Genomic Medicine Research, King Abdulaziz University, Jeddah, Saudi Arabia. ${ }^{2}$ Division of Neurosurgery, Department of Surgery, King Abdulaziz University Hospital, Jeddah, Saudi Arabia. ${ }^{3}$ King Fahad Medical Research Center, King Abdulaziz University, Jeddah, Saudi Arabia. ${ }^{4}$ Department of Surgery, Faculty of Medicine, King Abdulaziz University, Jeddah, Kingdom of Saudi Arabia. ${ }^{5}$ Department of Pathology, Faculty of Medicine, King Abdulaziz University Hospital, Jeddah, Saudi Arabia. ${ }^{6}$ Department of Pathology, King Faisal Specialist Hospital and Research Center, Jeddah, Saudi Arabia. ${ }^{7}$ Biochemistry Department, Faculty of Science, King Abdulaziz University, Jeddah, Saudi Arabia.

\section{Acknowledgements}

We thank Nadia Baqtian, Ohoud Subhi, Roa`a Al-Dayini, Fatima Al-Adwani, Manal Shabaat, Nawal Madkhali, Lobna S. Mira, Gladys J. Harder, Amal Hamed, Aisha Elaimi, and Shireen Hussain for excellent technical assistance.

\section{Competing interests}

The authors declare that they have no competing interests.

\section{Availability of data and materials}

The generated array data set has been deposited at the Gene Expression Omnibus (GEO) under Accession Number GSE100534. Other datasets generated and/or analyzed during the present study are available from the corresponding author on reasonable request.

\section{Consent for publication \\ Not applicable.}

\section{Ethics approval and consent to participate}

This research study was approved by the Research Ethics Committee of the King Abdulaziz University, Faculty of Medicine under Reference \#976-12. Written informed consent from the donors or the next of kin was obtained for the use of samples in research. 


\section{Funding}

This study was supported by King Abdulaziz City for Science and Technology (KACST) Grant AT-32-98.

\section{Publisher's Note}

Springer Nature remains neutral with regard to jurisdictional claims in published maps and institutional affiliations.

Received: 18 August 2017 Accepted: 18 December 2017 Published online: 29 December 2017

\section{References}

1. Venur VA, Leone JP. Targeted therapies for brain metastases from breast cancer. Int J Mol Sci. 2016;17:1543

2. Evans AJ, James JJ, Cornford EJ, Chan SY, Burrell HC, Pinder SE, Gutteridge E, Robertson JF, Hornbuckle J, Cheung KL. Brain metastases from breast cancer: identification of a high-risk group. Clin Oncol (R Coll Radiol) 2004;16:345-9.

3. Gaedcke J, Traub F, Milde S, Wilkens L, Stan A, Ostertag H, Christgen $\mathrm{M}$, von Wasielewski R, Kreipe HH. Predominance of the basal type and HER-2/neu type in brain metastasis from breast cancer. Mod Pathol. 2007:20:864-70.

4. Hicks DG, Short SM, Prescott NL, Tarr SM, Coleman KA, Yoder BJ, Crowe JP, Choueiri TK, Dawson AE, Budd GT, et al. Breast cancers with brain metastases are more likely to be estrogen receptor negative, express the basal cytokeratin CK5/6, and overexpress HER2 or EGFR. Am J Surg Pathol. 2006:30:1097-104.

5. Witzel I, Oliveira-Ferrer L, Pantel K, Muller V, Wikman H. Breast cancer brain metastases: biology and new clinical perspectives. Breast Cancer Res. 2016;18:8

6. Olson EM, Abdel-Rasoul M, Maly J, Wu CS, Lin NU, Shapiro CL. Incidence and risk of central nervous system metastases as site of first recurrence in patients with HER2-positive breast cancer treated with adjuvant trastuzumab. Ann Oncol. 2013;24:1526-33.

7. Murrell DH, Hamilton AM, Mallett CL, van Gorkum $\mathrm{R}$, Chambers AF, Foster PJ. understanding heterogeneity and permeability of brain metastases in murine models of her2-positive breast cancer through magnetic resonance imaging: implications for detection and therapy. Transl Oncol. 2015;8:176-84.

8. Brogi E, Murphy CG, Johnson ML, Conlin AK, Hsu M, Patil S, Akram M, Nehhozina T, Jhaveri KL, Hudis CA, Seidman AD. Breast carcinoma with brain metastases: clinical analysis and immunoprofile on tissue microarrays. Ann Oncol. 2011;22:2597-603.

9. Rostami R, Mittal S, Rostami P, Tavassoli F, Jabbari B. Brain metastasis in breast cancer: a comprehensive literature review. J Neurooncol. 2016;127:407-14

10. Weil RJ, Palmieri DC, Bronder JL, Stark AM, Steeg PS. Breast cancer metastasis to the central nervous system. Am J Pathol. 2005;167:913-20.

11. Lin NU, Bellon JR, Winer EP. CNS metastases in breast cancer. J Clin Oncol. 2004;22:3608-17.

12. Sirkisoon SR, Carpenter RL, Rimkus T, Miller L, Metheny-Barlow L, Lo HW. EGFR and HER2 signaling in breast cancer brain metastasis. Front Biosci (Elite Ed). 2016:8:245-63.

13. Leone Jé $P$, Leone BA. Breast cancer brain metastases: the last frontier. Exp Hematol Oncol. 2015:4:33.

14. Shonka N, Venur VA, Ahluwalia MS. Targeted treatment of brain metastases. Curr Neurol Neurosci Rep. 2017:17:37.

15. Ni J, Ramkissoon SH, Xie S, Goel S, Stover DG, Guo H, Luu V, Marco E, Ramkissoon LA, Kang YJ, et al. Combination inhibition of PI3K and mTORC1 yields durable remissions in mice bearing orthotopic patient-derived xenografts of HER2-positive breast cancer brain metastases. Nat Med. 2016;22:723-6.

16. Singh M, Manoranjan B, Mahendram S, McFarlane N, Venugopal C, Singh SK. Brain metastasis-initiating cells: survival of the fittest. Int J Mol Sci. 2014:15:9117-33.

17. Wilhelm I, Molnar J, Fazakas C, Hasko J, Krizbai IA. Role of the bloodbrain barrier in the formation of brain metastases. Int J Mol Sci. 2013;14:1383-411.
18. Kusters B, Kats G, Roodink I, Verrijp K, Wesseling P, Ruiter DJ, de Waal RM, Leenders WP. Micronodular transformation as a novel mechanism of VEGF-A-induced metastasis. Oncogene. 2007;26:5808-15.

19. Kienast $Y$, von Baumgarten $L$, Fuhrmann $M$, Klinkert WE, Goldbrunner $R$, Herms J, Winkler F. Real-time imaging reveals the single steps of brain metastasis formation. Nat Med. 2010;16:116-22.

20. Fidler IJ, Talmadge JE. Evidence that intravenously derived murine pulmonary melanoma metastases can originate from the expansion of a single tumor cell. Cancer Res. 1986:46:5167-71.

21. Brastianos PK, Carter SL, Santagata S, Cahill DP, Taylor-Weiner A, Jones RT, Van Allen EM, Lawrence MS, Horowitz PM, Cibulskis K, et al. Genomic characterization of brain metastases reveals branched evolution and potential therapeutic targets. Cancer Discov. 2015:5:1164-77.

22. Lee JY, Park K, Lee E, Ahn T, Jung HH, Lim SH, Hong M, Do IG, Cho EY, Kim $\mathrm{DH}$, et al. Gene expression profiling of breast cancer brain metastasis. Sci Rep. 2016:6:28623.

23. Momeny M, Saunus JM, Marturana F, McCart Reed AE, Black D, Sala G, lacobelli S, Holland JD, Yu D, Da Silva L, et al. Heregulin-HER3-HER2 signaling promotes matrix metalloproteinase-dependent blood-brain-barrier transendothelial migration of human breast cancer cell lines. Oncotarget. 2015;6:3932-46.

24. Bos PD, Zhang XH, Nadal C, Shu W, Gomis RR, Nguyen DX, Minn AJ, van de Vijver MJ, Gerald WL, Foekens JA, Massague J. Genes that mediate breast cancer metastasis to the brain. Nature. 2009;459:1005-9.

25. Sato R, Nakano T, Hosonaga M, Sampetrean O, Harigai R, Sasaki T, Koya I, Okano H, Kudoh J, Saya H, Arima Y. RNA sequencing analysis reveals interactions between breast cancer or melanoma cells and the tissue microenvironment during brain metastasis. Biomed Res Int. 2017:2017:8032910.

26. Chen Q, Boire A, Jin X, Valiente M, Er EE, Lopez-Soto A, Jacob LS, Patwa $\mathrm{R}$, Shah $\mathrm{H}$, Xu K, et al. Carcinoma-astrocyte gap junctions promote brain metastasis by cGAMP transfer. Nature. 2016;533:493-8.

27. Park ES, Kim SJ, Kim SW, Yoon SL, Leem SH, Kim SB, Kim SM, Park YY, Cheong JH, Woo HG, et al. Cross-species hybridization of microarrays for studying tumor transcriptome of brain metastasis. Proc Natl Acad Sci USA. 2011;108:17456-61.

28. Klein A, Olendrowitz C, Schmutzler R, Hampl J, Schlag PM, Maass $\mathrm{N}$, Arnold N, Wessel R, Ramser J, Meindl A, et al. Identification of brain- and bone-specific breast cancer metastasis genes. Cancer Lett. 2009:276:212-20

29. Salhia B, Kiefer J, Ross JT, Metapally R, Martinez RA, Johnson KN, DiPerna DM, Paquette KM, Jung S, Nasser S, et al. Integrated genomic and epigenomic analysis of breast cancer brain metastasis. PLOS ONE. 2014;9:e85448.

30. Nishizuka I, Ishikawa T, Hamaguchi Y, Kamiyama M, Ichikawa Y, Kadota K, Miki R, Tomaru Y, Mizuno Y, Tominaga N, et al. Analysis of gene expression involved in brain metastasis from breast cancer using cDNA microarray. Breast Cancer. 2002;9:26-32.

31. Palmieri D, Fitzgerald D, Shreeve SM, Hua E, Bronder JL, Weil RJ, Davis S, Stark AM, Merino MJ, Kurek R, et al. Analyses of resected human brain metastases of breast cancer reveal the association between up-regulation of hexokinase 2 and poor prognosis. Mol Cancer Res. 2009:7:1438-45.

32. Khaitan D, Sankpal UT, Weksler B, Meister EA, Romero IA, Couraud PO, Ningaraj NS. Role of KCNMA1 gene in breast cancer invasion and metastasis to brain. BMC Cancer. 2009;9:258.

33. McMullin RP, Wittner BS, Yang C, Denton-Schneider BR, Hicks D, Singavarapu R, Moulis S, Lee J, Akbari MR, Narod SA, et al. A BRCA1 deficientlike signature is enriched in breast cancer brain metastases and predicts DNA damage-induced poly (ADP-ribose) polymerase inhibitor sensitivity. Breast Cancer Res. 2014;16:R25.

34. Duchnowska R, Jarzab M, Zebracka-Gala J, Matkowski R, Kowalczyk A Radecka B, Kowalska M, Pfeifer A, Foszczynska-Kloda M, Musolino A, et al. Brain metastasis prediction by transcriptomic profiling in triple-negative breast cancer. Clin Breast Cancer. 2016;17:e65-75.

35. Schulten HJ, Hussein D, Al-Adwani F, Karim S, Al-Maghrabi J, Al-Sharif M Jamal A, Bakhashab S, Weaver J, Al-Ghamdi F, et al. Microarray expression profiling identifies genes, including cytokines, and biofunctions, as diapedesis, associated with a brain metastasis from a papillary thyroid carcinoma. Am J Cancer Res. 2016:6:2140-61. 
36. Merdad A, Karim S, Schulten HJ, Jayapal M, Dallol A, Buhmeida A, AlThubaity F, Gari IM, Chaudhary AG, Abuzenadah AM, Al-Qahtani MH. Transcriptomics profiling study of breast cancer from Kingdom of Saudi Arabia revealed altered expression of adiponectin and fatty acid binding protein 4: is lipid metabolism associated with breast cancer? BMC Genom. 2015;16(Suppl 1):S11.

37. Schulten HJ, Al-Mansouri Z, Baghallab I, Bagatian N, Subhi O, Karim S, Al-Aradati H, Al-Mutawa A, Johary A, Meccawy AA, et al. Comparison of microarray expression profiles between follicular variant of papillary thyroid carcinomas and follicular adenomas of the thyroid. BMC Genom 2015;16(Suppl 1):S7

38. Schulten HJ, Alotibi R, Al-Ahmadi A, Ata M, Karim S, Huwait E, Gari M, Al-Ghamdi K, Al-Mashat F, Al-Hamour O, et al. Effect of BRAF mutational status on expression profiles in conventional papillary thyroid carcinomas. BMC Genom. 2015;16(Suppl 1):S6.

39. Robinson MD, Speed TP. A comparison of affymetrix gene expression arrays. BMC Bioinform. 2007;8:449.

40. Tuominen VJ, Ruotoistenmäki S, Viitanen A, Jumppanen M, Isola J. ImmunoRatio: a publicly available web application for quantitative image analysis of estrogen receptor (ER), progesterone receptor (PR), and Ki-67. Breast Cancer Res. 2010;12:R56.

41. Afgan E, Baker D, van den Beek M, Blankenberg D, Bouvier D, Cech M, Chilton J, Clements D, Coraor N, Eberhard C, et al. The Galaxy platform for accessible, reproducible and collaborative biomedical analyses: 2016 update. Nucleic Acids Res. 2016;44:W3-10.

42. Blankenberg D, Gordon A, Von Kuster G, Coraor N, Taylor J, Nekrutenko A. Manipulation of FASTQ data with galaxy. Bioinformatics. 2010;26:1783-5.

43. Li H, Durbin R. Fast and accurate short read alignment with BurrowsWheeler transform. Bioinformatics. 2009;25:1754-60.

44. Garrison E MG: Haplotype-based variant detection from short-read sequencing. In Preprint at arXiv:12073907v2 [q-bioGN]. 2012.

45. Flanagan SE, Patch AM, Ellard S. Using SIFT and PolyPhen to predict loss-of-function and gain-of-function mutations. Genet Test Mol Biomark. 2010;14:533-7.

46. Sherry ST, Ward MH, Kholodov M, Baker J, Phan L, Smigielski EM, Sirotkin K. dbSNP: the NCBI database of genetic variation. Nucleic Acids Res. 2001;29:308-11.

47. Forbes SA, Tang G, Bindal N, Bamford S, Dawson E, Cole C, Kok CY, Jia M, Ewing $\mathrm{R}$, Menzies $\mathrm{A}$, et al. COSMIC (the catalogue of somatic mutations in cancer): a resource to investigate acquired mutations in human cancer. Nucleic Acids Res. 2010;38:D652-7.

48. Schulten HJ, Salama S, Al-Mansouri Z, Alotibi R, Al-Ghamdi K, Al-Hamour $\mathrm{OA}$, Sayadi $\mathrm{H}$, Al-Aradati $\mathrm{H}, \mathrm{Al}$-Johari $\mathrm{A}$, Huwait $\mathrm{E}$, et al. BRAF mutations in thyroid tumors from an ethnically diverse group. Hered Cancer Clin Pract. 2012;10:10.

49. Lafontaine DL, Tollervey D. Birth of the snoRNPs: the evolution of the modification-guide snoRNAs. Trends Biochem Sci. 1998;23:383-8.

50. Nallar SC, Kalvakolanu DV. Regulation of snoRNAs in cancer: close encounters with interferon. J Interferon Cytokine Res. 2013;33:189-98.

51. Stepanov GA, Filippova JA, Komissarov AB, Kuligina EV, Richter VA, Semenov DV. Regulatory role of small nucleolar RNAs in human diseases. Biomed Res Int. 2015;2015:206849.

52. Falaleeva M, Pages A, Matuszek Z, Hidmi S, Agranat-Tamir L, Korotkov K, Nevo Y, Eyras E, Sperling R, Stamm S. Dual function of C/D box small nucleolar RNAs in rRNA modification and alternative pre-mRNA splicing. Proc Natl Acad Sci USA. 2016;113:E1625-34.

53. Herter EK, Stauch M, Gallant M, Wolf E, Raabe T, Gallant P. snoRNAs are a novel class of biologically relevant Myc targets. BMC Biol. 2015;13:25.

54. Schramm A, Koster J, Marschall T, Martin M, Schwermer M, Fielitz K, Buchel G, Barann M, Esser D, Rosenstiel P, et al. Next-generation RNA sequencing reveals differential expression of MYCN target genes and suggests the mTOR pathway as a promising therapy target in MYCNamplified neuroblastoma. Int J Cancer. 2013;132:E106-15.

55. Su H, Xu T, Ganapathy S, Shadfan M, Long M, Huang TH, Thompson I, Yuan ZM. Elevated snoRNA biogenesis is essential in breast cancer. Oncogene. 2014;33:1348-58.

56. Krishnan P, Ghosh S, Wang B, Heyns M, Graham K, Mackey JR, Kovalchuk O, Damaraju S. Profiling of small nucleolar RNAs by next generation sequencing: potential new players for breast cancer prognosis. PLoS ONE. 2016;11:e0162622.
57. Kim JW, Kim JY, Kim JE, Kim SK, Chung HT, Park CK. HOXA10 is associated with temozolomide resistance through regulation of the homologous recombinant DNA repair pathway in glioblastoma cell lines. Genes Cancer. 2014;5:165-74.

58. Milanovic D, Sticht C, Rohrich M, Maier P, Grosu AL, Herskind C. Inhibition of 13-cis retinoic acid-induced gene expression of reactive-resistance genes by thalidomide in glioblastoma tumours in vivo. Oncotarget. 2015;6:28938-48

59. Dupuis-Sandoval F, Poirier M, Scott MS. The emerging landscape of small nucleolar RNAs in cell biology. Wiley Interdiscip Rev RNA. 2015;6:381-97.

60. Blenkiron C, Hurley DG, Fitzgerald S, Print CG, Lasham A. Links between the oncoprotein YB-1 and small non-coding RNAs in breast cancer. PLoS ONE. 2013;8:e80171.

61. Liang F, Qu H, Lin Q, Yang Y, Ruan X, Zhang B, Liu Y, Yu C, Zhang H, Fang X, Hao $X$. Molecular biomarkers screened by next-generation RNA sequencing for non-sentinel lymph node status prediction in breast cancer patients with metastatic sentinel lymph nodes. World J Surg Oncol. 2015:13:258.

62. Lafontaine DL. Noncoding RNAs in eukaryotic ribosome biogenesis and function. Nat Struct Mol Biol. 2015;22:11-9.

63. Chu L, Su MY, Maggi LB Jr, Lu L, Mullins C, Crosby S, Huang G, Chng WJ, Vij $\mathrm{R}$, Tomasson $\mathrm{MH}$. Multiple myeloma-associated chromosomal translocation activates orphan snoRNA ACA11 to suppress oxidative stress. J Clin Invest. 2012;122:2793-806.

64. Gridasova AA, Henry RW. The p53 tumor suppressor protein represses human snRNA gene transcription by RNA polymerases II and III independently of sequence-specific DNA binding. Mol Cell Biol. 2005;25:3247-60.

65. Wu J, Lu LY, Yu X. The role of BRCA1 in DNA damage response. Protein Cell. 2010;1:117-23.

66. Wiegmans AP, Al-Ejeh F, Chee N, Yap PY, Gorski JJ, Silva LD, Bolderson E, Chenevix-Trench G, Anderson R, Simpson PT, et al. Rad51 supports triple negative breast cancer metastasis. Oncotarget. 2014;5:3261-72.

67. Woditschka S, Evans L, Duchnowska R, Reed LT, Palmieri D, Qian Y, Badve S, Sledge G Jr, Gril B, Aladjem Ml, et al. DNA double-strand break repair genes and oxidative damage in brain metastasis of breast cancer. J Natl Cancer Inst. 2014;106:dju145.

68. Liu Y, Burness ML, Martin-Trevino R, Guy J, Bai S, Harouaka R, Brooks MD, Shang L, Fox A, Luther TK, et al. RAD51 mediates resistance of cancer stem cells to PARP inhibition in triple-negative breast cancer. Clin Cancer Res. 2017:23:514-22

69. Eelen G, Vanden Bempt I, Verlinden L, Drijkoningen M, Smeets A, Neven P, Christiaens MR, Marchal K, Bouillon R, Verstuyf A. Expression of the BRCA1-interacting protein Brip1/BACH1/FANCJ is driven by E2F and correlates with human breast cancer malignancy. Oncogene. 2008;27:4233-41.

70. Gnad F, Doll S, Manning G, Arnott D, Zhang Z. Bioinformatics analysis of thousands of TCGA tumors to determine the involvement of epigenetic regulators in human cancer. BMC Genom. 2015;16:S5.

71. Tin AS, Sundar SN, Tran KQ, Park AH, Poindexter KM, Firestone GL. Antiproliferative effects of artemisinin on human breast cancer cells requires the downregulated expression of the E2F1 transcription factor and loss of E2F1-target cell cycle genes. Anticancer Drugs. 2012;23:370-9.

72. Pujana MA, Han JD, Starita LM, Stevens KN, Tewari M, Ahn JS, Rennert G, Moreno V, KirchhoffT, Gold B, et al. Network modeling links breast cancer susceptibility and centrosome dysfunction. Nat Genet. 2007;39:1338-49.

73. Priedigkeit N, Hartmaier RJ, Chen Y, Vareslija D, Basudan A, Watters RJ, Thomas R, Leone JP, Lucas PC, Bhargava R, et al. Intrinsic subtype switching and acquired ERBB2/HER2 amplifications and mutations in breast cancer brain metastases. JAMA Oncol. 2016;3:666-71.

74. Chandriani S, Frengen E, Cowling VH, Pendergrass SA, Perou CM, Whitfield $M L$, Cole MD. A core MYC gene expression signature is prominent in basal-like breast cancer but only partially overlaps the core serum response. PLoS ONE. 2009;4:e6693.

75. Terunuma A, Putluri N, Mishra P, Mathe EA, Dorsey TH, Yi M, Wallace TA, Issaq HJ, Zhou M, Killian JK, et al. MYC-driven accumulation of 2-hydroxyglutarate is associated with breast cancer prognosis. J Clin Invest. 2014;124:398-412.

76. Lopez-Garcia C, Sansregret L, Domingo E, McGranahan N, Hobor S, Birkbak NJ, Horswell S, Gronroos E, Favero F, Rowan AJ, et al. BCL9L dysfunction impairs caspase-2 expression permitting aneuploidy tolerance in colorectal cancer. Cancer Cell. 2017;31:79-93. 
77. Hansford S, Kaurah P, Li-Chang H, Woo M, Senz J, Pinheiro H, Schrader KA, Schaeffer DF, Shumansky K, Zogopoulos G, et al. Hereditary diffuse gastric cancer syndrome: $\mathrm{CDH} 1$ mutations and beyond. JAMA Oncol. 2015;1:23-32

78. Jiao S, Li N, Cai S, Guo H, Wen Y. Inhibition of CYFIP2 promotes gastric cancer cell proliferation and chemoresistance to 5-fluorouracil through activation of the Akt signaling pathway. Oncol Lett. 2017;13:2133-40.

79. Liu B, Shats I, Angus SP, Gatza ML, Nevins JR. Interaction of E2F7 transcription factor with E2F1 and C-terminal-binding protein (CtBP) provides a mechanism for E2F7-dependent transcription repression. J Biol Chem. 2013;288:24581-9.

80. Singh P, Ravanan P, Talwar P. Death associated protein kinase 1 (DAPK1): a regulator of apoptosis and autophagy. Front Mol Neurosci. 2016;9:46.

81. Sherman JH, Karpinski BA, Fralish MS, Cappuzzo JM, Dhindsa DS, Thal AG, Moody SA, LaMantia AS, Maynard TM. Foxd4 is essential for establishing neural cell fate and for neuronal differentiation. Genesis. 2017;55:e23031.

82. Klein SL, Neilson KM, Orban J, Yaklichkin S, Hoffbauer J, Mood K, Daar IO, Moody SA. Conserved structural domains in FoxD4L1, a neural forkhead box transcription factor, are required to repress or activate target genes. PLOS ONE. 2013;8:e61845.

83. Shao H, Gu Y, Ding J, Lu P, Ruan T, Lu W. HEPACAM inhibited the growth and migration of cancer cells in the progression of non-small cell lung cancer. Tumour Biol. 2016;37:2621-7.
84. Fry MJ. Phosphoinositide 3-kinase signalling in breast cancer: how big a role might it play? Breast Cancer Res. 2001;3:304-12.

85. Padua D, Zhang XH, Wang Q, Nadal C, Gerald WL, Gomis RR, Massague J. TGFbeta primes breast tumors for lung metastasis seeding through angiopoietin-like 4. Cell. 2008;133:66-77.

86. Wakefield A, Soukupova J, Montagne A, Ranger J, French R, Muller WJ, Clarkson RW. Bcl3 selectively promotes metastasis of ERBB2-driven mammary tumors. Cancer Res. 2013;73:745-55.

87. Hervouet E, Claude-Taupin A, Gauthier T, Perez V, Fraichard A, Adami P, Despouy G, Monnien F, Algros MP, Jouvenot M, et al. The autophagy GABARAPL1 gene is epigenetically regulated in breast cancer models. BMC Cancer. 2015;15:729.

88. Eckerdt F, Yuan J, Strebhardt K. Polo-like kinases and oncogenesis. Oncogene. 2005;24:267-76.

89. Lund PJ, Elias JE, Davis MM. Global Analysis of O-GICNAc glycoproteins in activated human T cells. J Immunol. 2016;197:3086-98.

90. Zhao B, Zong G, Xie Y, Li J, Wang H, Bian E. Downregulation of ubiquitinassociated protein 2-like with a short hairpin RNA inhibits human glioma cell growth in vitro. Int J Mol Med. 2015;36:1012-8.

91. Pons DG, Nadal-Serrano M, Torrens-Mas M, Valle A, Oliver J, Roca P. UCP2 inhibition sensitizes breast cancer cells to therapeutic agents by increasing oxidative stress. Free Radic Biol Med. 2015;86:67-77.

\section{Submit your next manuscript to BioMed Central and we will help you at every step:}

- We accept pre-submission inquiries

- Our selector tool helps you to find the most relevant journal

- We provide round the clock customer support

- Convenient online submission

- Thorough peer review

- Inclusion in PubMed and all major indexing services

- Maximum visibility for your research

Submit your manuscript at www.biomedcentral.com/submit
() Biomed Central 